\title{
Single-cell RNA sequencing identifies heterogeneous cell subtypes within gingival tissue
}

\section{Shujiao Qian}

Shanghai Ninth People's Hospital

Qianru Huang

Shanghai Institute of Immunology

Junyu Shi

Shanghai Ninth People's Hospital

Linyi Zhou

Shanghai Ninth People's Hospital

Yi Zhao

Shanghai Institute of Immunology

\section{Bin Li}

Shanghai Institute of Immunology https://orcid.org/0000-0002-7640-8884

Hongchang Lai ( $\sim$ hongchanglai@126.com )

Shanghai Ninth People's Hospital

\section{Article}

Keywords: single-cell sequencing, gingival, periodontitis

Posted Date: November 6th, 2020

DOI: https://doi.org/10.21203/rs.3.rs-101475/v1

License: (c) (i) This work is licensed under a Creative Commons Attribution 4.0 International License.

Read Full License 


\section{Single-cell RNA sequencing identifies heterogeneous cell subtypes within gingival tissue}

$9 \quad$ *Authors contributing equally as co-first authors.

10 \# Correspondence authors. Hong-chang Lai, 1hc9@ hotmail.com and Bin Li, binli@shsmu.edul.cn

12 'Department of Implant Dentistry, Shanghai Ninth Peoples' Hospital, College of Stomatology, 13 Shanghai Jiao Tong University School of Medicine, National Clinical Research Center for Oral

14 Diseases, Shanghai Key Laboratory of Stomatology \& Shanghai Research Institute of

15 Stomatology, Shanghai, 200011, China

16 2Shanghai Institute of Immunology, Shanghai Jiao Tong University School of Medicine,

17 Shanghai 200025, China

$18{ }^{3}$ Department of Immunology and Microbiology, Shanghai Jiao Tong University School of

19 Medicine, Shanghai 200025, China

\section{Conflict of interest and source of funding statements}

22 None of the authors have conflict of interest. This study has been supported by The Project of Biobank

23 from Shanghai Ninth People's Hospital, Shanghai Jiao Tong University School of Medicine

24 (YBKA201906).

25

26 
Abstract

Owing to the high prevalence of periodontitis and its impact on quality of life, a rigorous characterization of periodontal tissue is necessary. However, this has previously been impeded by the cellular heterogeneity of periodontal tissue. Here, we characterized the gene expression of gingival tissue in health and periodontitis at single-cell resolution. We reported the loss of epithelial homeostasis-associated genes expressed by specific epithelial cell types in periodontitis. We detected endothelial cells highly associated with immune regulation and fibroblasts potentially involved in bone remodeling. We revealed the myeloid cells with diverse clusters dominated by pro-inflammatory phenotype. We further identified cell subtypes specifically expressing periodontitis related genes and highlighted the cell-cell communication alteration between conditions. This novel single-cell study generated a comprehensive catalog of cells types and interaction networks in the human gingiva for the first time, thus offering deeper insights into the biological foundation of periodontal homeostasis and diseases, which will be helpful in advancing periodontal diagnosis and therapy.

42 Keywords single-cell sequencing, gingival, periodontitis

\section{Introduction}

Periodontium is an umbrella term for tissues that surround and support the teeth, including soft tissues (gingiva and periodontal ligament) and hard tissues (alveolar bone and cementum) $)^{1,2}$. Periodontitis, a chronic or aggressive inflammatory lesion of the collective periodontium, is characterized by irreversible and progressive degradation of the periodontal tissue and causes tooth loss and alveolar bone defects. Current evidence has demonstrated independent associations between periodontitis and several non-communicable diseases including cardiovascular disease, diabetes, chronic kidney disease, respiratory diseases, and cognitive disorders ${ }^{3,4}$. Being the sixth most common human disease, severe periodontitis affects more than one tenth of the global adult population, hence representing a substantial health and socioeconomic burden ${ }^{5}$. Notwithstanding the fact that inflammation is initiated by bacteria residing in the biofilm at gingival or oral mucosal surface, intrinsic host factors and environmental stressors determine the periodontal inflammatory response ${ }^{6}$. 
As the major constituents of periodontal soft tissue, gingiva are the first layer of defense against oral microorganisms ${ }^{7}$. Several types of cells are arrayed in an orderly manner in gingiva including epithelial cells (gingival, sulcular, junctional, and enamel), blast cells (fibroblast, cementoblasts, ameloblasts and osteoblasts), immune cells (neutrophils, T cells, B cells, monocytes, macrophage and dendritic cells), endothelial cells and neural cells, and undifferentiated mesenchymal cells ${ }^{8-10}$. Emerging evidence has indicated that structural cells, such as fibroblast, as well as T cells and macrophages in gingiva, are highly heterogeneous ${ }^{11-13}$,

64 leading, to various degrees, to periodontal inflammatory response. Nowadays, the diversity of cell subsets has been recognized as a substrate for new therapeutic strategies. For instance, the Treg-recruiting formulation system has been injected to treat severe experimental periodontitis ${ }^{14}$. Agents inducing the inflammatory-to-resolving conversion of macrophages were suggested to arrest periodontitis progression and stimulate bone regeneration ${ }^{15}$. However, the heterogeneity of in situ cells under periodontal health and disease conditions remain largely unknown.

On the other hand, studies have been performed to offer a global overview of molecular events of the inflammatory response in both in vivo and in vitro models. Multiple pathways were

72 reported to be activated in in vitro infectious models, including inflammatory cytokines, reactive oxygen species generation, free radical scavenging, cell cycle, host defense mechanisms and 74 Protein Kinase B/mitogen-activated protein kinase signaling pathway ${ }^{16-21}$. Studies of in vivo 75 models, which focused on biopsy samples from periodontitis patients, have also demonstrated 76 that genes in apoptosis, antimicrobial humoral response, antigen presentation, and regulation of 77 metabolic process pathways were highly involved ${ }^{22}$.Transcriptome analysis based on nextgeneration sequencing technology, especially RNA-seq, has revealed novel gene expression and splicing patterns in gingival biopsies from periodontitis patients compared with those from the

80 healthy controls, such as upregulation of genes in the pathways of defense/immunity protein, 81 receptor, protease, and signaling molecules, and downregulation in cytoskeletal and structural 82 proteins ${ }^{23}$. Horie et al. performed transcriptome analysis of gingival fibroblast from periodontitis 83 patients and identified osteogenic markers DLX5 and RUNX2 long variant as novel regulators ${ }^{24}$. 84 Lundmark et al. provided the gene expression profiles of the periodontitis affected gingival 85 biopsies, and found the inflamed area had upregulated expression of IGLL5, SSR4, MZB1 and 86 XBP1, compared to the non-inflamed area ${ }^{25}$. 
Although transcriptome analysis based on bulk tissue RNA-seq has provided comprehensive gene expression profiles on paired gingival biopsies from both periodontitis-affected and healthy tissues, most studies only focused on the averaged transcriptional signatures on a preselected cell type or crossed all cell types in the whole tissue without the information on the cellular heterogeneity in the periodontal tissue. Herein, we reported the transcriptomic profiling of a total of 29,967 single cells of human gingival tissues from 2 patients with periodontitis and 2 healthy donors. We identified specific epithelial cell types associated with compromised epithelial

94 defense in periodontitis. We detected endothelial cells highly associated with immune regulation and fibroblasts potentially involved in bone remodeling. We revealed the myeloid cells with diverse clusters dominated by pro-inflammatory phenotype. We further identified cell subtypes specifically expressing periodontitis related genes and investigated the cell-cell communication alteration between conditions. Overall, our study describes a transcriptome profile of human healthy and diseased gingival tissue at the single-cell resolution. It would offer a novel perspective on the periodontal inflammatory response and serve as a useful resource for the scientific community.

\section{Results}

\section{scRNA-seq and cell typing of gingival tissues under healthy and disease conditions}

To generate transcriptome profiles of human gingival tissues, samples from four donors were obtained. Two of the donors were diagnosed with periodontitis (P1 and P2). The other two samples were obtained from healthy volunteers who underwent crown lengthening procedures. Tissues were collected fresh, dissected, and digested into single cells (Figure 1A, Supplementary Table 1). For each sample, single cells were captured using the droplet-based microfluidic system Chromium (10X Genomics). The number of genes expressed differed in the various cell 111 types, particularly between immune and non-immune cells (Supplementary Figure 1A and 1B). Considering potential batch effects and background noises among samples, we applied

114 method in Seurat ${ }^{27}$. The single-cell data are presented in two-dimensional space using a uniform 115 manifold approximation and projection (UMAP) method (Figure 1B). Clustering

116 analysis cataloged these cells into ten distinct cell lineages annotated with canonical marker gene 117 expression, thereby corresponding to epithelial cells, stromal cells (endothelial, vascular mural 
118 and fibroblast), and immune cells (T, NK, B, plasma, myeloid and mast cells) (Figure 1C).

119 Differential expressed genes (DEGs) of each cell type were computed and the top 5 DEGs were 120 visualized (Supplementary Figure 1C).

121 Then we analyzed the distribution of the different cell lineages that accumulated among

122 various conditions (Figure 1D). In healthy tissues, the epithelial cell has the largest proportion

123 which comprises nearly $92 \%$ of all cells. However, in disease tissues, it only comprises $4.6 \%$. In

124 contrast, all the other cell types have their proportion increased in disease tissues, especially

125 immune cells (T, B and plasma cells), which is consistent with the inflammation phenotype.

126 Thus, although it was expected that immune activation, including the infiltration of the gingival

127 tissue by $\mathrm{T}$ and $\mathrm{B}$ cells, would be involved in the pathogenesis, we provided a quantified

128 description of the proportion change in the cell types. Our result also indicated that due to the

129 enormous change in cell proportion, bulk RNA sequencing would be a less ideal method to

130 measure gene expression changes when comparing healthy and periodontitis tissues, since the

131 results would be largely confounded by cell proportion.

132 Next, we assessed the cell type-specific expression patterns of genes related to Mendelian

133 disorders (based on OMIM database), which provided insights into the contribution of specific

134 cell types to gingival abnormality (Figure 1E and Supplementary Table 2). Cell type-specific

135 expression patterns confirmed fibroblast as particularly high expressors of the $C 1 S$ and $C 1 R$

136 gene, mutated in Ehlers-Danlos syndrome, periodontal type. Two reported gene mutants, SOS1 ${ }^{28}$

137 and $R E S T^{29}$, associated with gingival fibromatosis (GF), were highly expressed in endothelial

138 cells. Additionally, the CTSC gene, mutations of which are responsible for aggressive

139 periodontitis in juveniles, was found to be widely expressed in the non-immune cell population.

140 While in immune cells, the expression of CTSC was highly enriched in myeloid cells.

\section{Compromised epithelial barrier defense in periodontitis}

143 The gingival epithelium, which consists of oral gingival epithelium, sulcular epithelium, 144 and junctional epithelium, plays a vital role against bacterial invasion. We partitioned the

145 epithelial cells into four diverse clusters based on previous reports ${ }^{30,31}$ : junctional epithelium

146 (JE1-JE2), basal (BAS1-BAS2), spinous (SPN1-SPN3), and differentiated granular keratinocytes

147 (GRN1-GRN3) (Figure 2A, B). JE2 and JE1 dominated the epithelial cells in periodontitis, with 148 significantly reduced GRN1/GRN2 and completely disappeared SPN1/SPN2 (Supplementary 
149 Figure 2A). Immunohistochemical showed the outermost layer of the epithelium, referring to 150 GRN and SPN, experienced severe ruptures in patients (Figure 2C). These data showed that the 151 integrity of epithelial tissue is seriously damaged in periodontitis compared with the healthy 152 controls.

153 We further analyzed the expression of characteristic genes and top 5 DEGs in each cluster 154 (Figure 2D and Supplementary Figure 2B). For example, JE1 express amelogenesis associated 155 proteins ODAM and ODAPH (C4orf26), which may participate in enamel mineralization.

156 Meanwhile, we found both JE1 and JE2 expressed serum amyloid A family (SAA1 and SAA2), 157 which can induce pathogenic Th17 and promote inflammation in experimental allergic 158 encephalomyelitis and inflammatory bowel disease ${ }^{32}$. Besides, HLA-DRs (HLA-DRB1 and 159 HLA-DRA) presenting extracellular pathogens were found highly expressed in JE2 and BAS1.

160 BAS2 exhibited high levels of mitosis-related genes, denoting its possible stage at $\mathrm{G} 2 / \mathrm{m}$ stages

161 (Supplementary Figure 2C). The three spinous clusters were found to express GSTA4 involved in 162 anti-oxidative stress $^{33}$ and $C Y B 5 A$ detoxifying carcinogens from cigarette ${ }^{34}$, thus playing 163 essential roles in the detoxification of harmful foreign chemicals. GRN2 and GRN3 expressed a 164 group of epidermal differentiation complex genes, which are essential for terminal differentiation 165 of the human epidermis. Meanwhile, the two clusters expressed genes associated with the 166 inflammatory response. We also noticed that GRN1-3 and JE1 expressed $\beta$-defensins (DEFB1) 167 and S100A7/8/9 against bacterial challenge, underscoring their role as the outermost layer in 168 direct contact with the environment. Together, these data suggested that the damaged integrity of 169 the epithelium barrier, leading to the loss of epithelial homeostasis-associated genes expressed 170 by particular cell clusters in periodontitis, may constitute an important mechanism for 171 periodontal pathogenicity. Analysis of the pathway in epithelial subsets was performed by gene 172 set variation analysis (GSVA) ${ }^{35}$ (Supplementary Figure 2D). The enriched terms highlight the 173 difference in clusters and support the above inference about the function of each cell type, such 174 as amelogenesis for JE1, DNA replication for BAS2, and keratinocyte differentiation for GRN3. 175 SCENIC ${ }^{36}$ was utilized to identify different transcription factors (TFs) underlying the 176 regulation of each epithelial phenotype (Figure 2E and Supplementary Table 3). For instance, 177 Runx2 might be a potential regulator of the ODAM expression in junctional epithelium, thus 178 playing a critical role in maintaining the integrity of the dentogingival junction ${ }^{37,38}$. Next, we 179 used Monocle $2^{40,41}$ to further understand the relationship between epithelial cell states. 
180 Combining trajectory plot and pseudotime analysis, we can form conclusions about the

181 conditions and relationships among these clusters (Figure 2F). We found junctional epithelial

182 and basal are both the beginning of the trajectory. This finding could be explained by the fact

183 that junctional epithelial has the basement membrane-like structures with low differentiation and

184 high regeneration ability ${ }^{42,43}$. Then, the trajectory experiences the spinous and end at the GRN3.

185 We found 487 genes that vary significantly along pseudotime (Supplementary Table 4), and the

186 heatmap showed the changes of expression pattern of 30 genes during the different stages

187 (Figure 2G). The markers used for the subgroup definition, such as FDCSP, KRT5, and SLURP1,

188 are indeed distributed along pseudotime trajectory. Some novel genes are specifically expressed

189 in different stages, such as reversible epithelial-mesenchymal transition associated CYR61 in JE ${ }^{44}$

190 and epidermal differentiation associated CALML5 in $\mathrm{GRN}^{45}$. This analysis may provide us

191 deeper insights into the development of epithelial clusters that will be helpful in advancing

192 periodontitis treatment and gingival tissue regeneration.

\section{Endothelial cells highly associated with immune regulation and fibroblasts potentially} involved in bone remodeling

To gain more insight into the heterogeneity of the gingival tissue, next, we focused on other non-immune clusters. The 1959 endothelial cells can be classified into three distinct subpopulations (Figure 3A). Endo_1 expressed ACKR1, SELE, and SELP. Endo_2 was characterized by GJA4, HEY1, and NOTCH4. We discovered a small group of lymphatic

201 (Figure 3B). The pathway analysis illustrates that Endo_1 is highly correlated with the immune 202 response, response to interferon gamma, and upregulating adhesion molecules to tether or roll 203 leukocyte. Meanwhile, Endo_1 is also involved in the regulation of blood pressure. In Endo_2, 204 Notch signaling and other molecules contributing to endothelium development and migration 205 were enriched. The enrichment pathways of Endo_3 meet the definition of LECs and are also 206 involved in cell substrate adhesion (Figure 3C). Consistent with previous studies, we identified 207 an epithelial cell state with high expression level of MHC class II genes such as HLA-DRA, $208 H L A-D R B 1$, and $H L A-D P B 1$, which was normally found in professional antigen-presenting 209 cells $^{47}$. The endothelial cell states in healthy tissue, particularly Endo_1, exhibited lower feature 210 score of MHC class II compared with those in diseased tissues, suggesting the importance of 
211 endothelial cells in gingival tissue-specific immunity (Figure 3D). Immunofluorescence assays

212 for the MHC class II marker HLA-DR and the endothelial cell marker PECAM1 (CD31) further

213 confirmed the existence of antigen-presenting endothelial cells in gingival tissues of periodontitis

214 (Figure 3E).

215 Gingival fibroblasts have long been recognized as a heterogeneous population, but the extent

216 of heterogeneity has hitherto remained poorly explored ${ }^{48}$. We identified two subclusters with

217 multiple differentially expressed genes against each other (Figure 4A). Fibro_1 were

218 characterized by high expression of CXCL13, IL32, and SFRP2, which are associated with B cell

219 recruitment or pro-inflammatory ${ }^{49,50}$. Fibro_2 expressed higher levels of OGN, PRELP, and

220 RUNX2 compared with Fibro_1 (Figure 4B). Notably, forced OGN expression can increase the

221 expression of RUNX2 and OCN, resulting in increased bone mass ${ }^{51}$, while PRELP expressed in

222 cartilage and basement membranes can impair osteoclastogenesis by inhibiting NF-kB ${ }^{52}$. Then

223 we compared pathway enrichment between two fibroblast clusters (Figure 4C). Fibro_1 showed

224 increased immune response pathways. Pathways associated with osteoblast development and

225 bone remodeling are increased in Fibro_2. With immunofluorescent staining, we confirmed that

226 OGN was detected in only a subset of fibroblast cells, which were labeled by anti-decorin

227 staining. However, the role of Fibro_2 in bone remodeling needs to be further verified. Finally,

228 we utilized $\mathrm{scHCL}^{47}$ to verify the cluster identification and further explore the similarities

229 between clusters (Figure 4E). By calculating Pearson correlation coefficients, the reliability of

230 the cluster identification was confirmed. Interestingly, both epithelial and fibroblast cells were

231 highly similar to those of esophageal origin, suggesting the effect of food intake on the cellular

232 function of gingival fibroblast and epithelial cells.

234 Diverse immune cell subtypes with hyper-inflammatory response in periodontitis

235 Most of the tissue destruction in periodontitis is determined by the host immune response.

236 To gain more insight into the host inflammatory and immune mechanisms in the gingival tissue,

237 we performed sub-clustering on myeloid cell types containing 148 cells from healthy donors and

238539 cells from patients. We revealed seven subtypes of myeloid cells ${ }^{53}$ (Figure 5A). Three DC

239 subsets were characterized by low expression of CD14, and three CD14-high expressing clusters

240 were identified as macrophages based on their high expression of CD68, CD163, and MRC1 ${ }^{53,54}$

241 (Supplementary Figure 3A). Plasmacytoid DC (pDC), cDC1, and cDC2 were further 
242 distinguished by specific expression of LILRA4/GZMB/JCHAIN, BATF3/CLEC9A/CADM1,

243 CDIC/CLEC1OA/FCERIA. Three clusters of CD14-high macrophages, Macro_PRDM1,

244 Macro_NLRP3, and Macro_C1QA, were distinguished based on the expression of

245 FCGR2B/PRDM1/HES1, NLRP3/IL1B/EREG, and C1QA/SEPP1/SPOE. There is a CD14+

246 monocyte cluster showing different features with DC and macrophages. All myeloid subtypes

247 could be found in both normal and patient samples (Figure 5B). Differentially expressed marker

248 genes between health and periodontitis were identified within each cell cluster (Figure 5C). The

249 pDC group highly expressed Granzyme B, which has been reported to suppress T-cell

250 expansion $^{55}$. Expression of the pro-inflammatory cytokine gene IL1B was relatively high in

251 Macro_PRDM1 and Macro_NLRP3. Further, Macro_NLRP3 highly expressing NLRP3 and

252 S100A8 showed a strong pro-inflammatory phenotype. Macro_PRDM1 in periodontitis

253 expressed a higher level of HES1, a transcription repressor controlling production of

254 macrophage-derived chemokines in inflammatory arthritis ${ }^{56}$ (Figure 5C).

255 The phenotypes of macrophages were analyzed in depth from angiogenesis and phagocytosis

256 (Figure 5D). The gene associated with phagocytosis was highly expressed in Macro_C1QA,

257 while Macro_NLRP3 dominated angiogenesis. Then we used RNA velocity ${ }^{57}$ to study the

258 developmental lineages of macrophages and projected the result onto the UMAP plot. With

259 Macro_PRDM1 at the intermediate stage, macrophages in gingival tissue intend to differentiate

260 from Macro_C1QA to pro-inflammatory Macro_NLRP3 phenotype. Besides, Macro_PRDM1

261 simultaneously resembled the signatures of M1 and M2 cells (Supplementary Figure 3B),

262 indicating that the dichotomous concept of macrophage function was inappropriate ${ }^{58}$.

263 Meanwhile, monocle2 was used to reveal the similar developmental lineages of macrophages,

264 and the heatmap showed the top genes associated with latent time (Supplementary Figure 3C and

265 3D). Osteoclast differentiation-related protein CCL3 and CXCL2 ${ }^{59}$, but not other chemokine

266 family genes, were found to be expressed in all macrophage subtypes in our data (Supplementary

267 Figure 3E), suggesting their role in the regulation of macrophage-osteoclast differentiation.

268 Another diverse immune cell cluster is $\mathrm{T}$ and NK cells, which were divided into five

269 subtypes (Figure 5E and 5G). CD4_CTLA4 highly express Treg-associated molecules, including

270 TNFRSF18, TNFRSF4, CTLA4, and FOXP3 (Supplementary Figure 3F). CD4_FOS from

271 patients highly expressed immediate-early genes $(F O S, J U N)$, which may be associated with $\mathrm{T}$

272 cell activation or the effect of enzymatic digestion ${ }^{60}$. Besides, CD8 and NK express cytotoxic 
genes, including GZMK, GZMA, GNLY, and so on. Notably, a higher expression level of CCR5

274 or CCR1 ligand CCLA/CCL4L2/CCL3L3 by CD8 T cells was observed in patients, underscoring their role in inflammatory cell recruitment in periodontitis.

\section{Differentially expressed genes and cell-cell interactions between periodontal health and}

\section{disease conditions}

Based on the previous bulk RNA sequencing ${ }^{23,25,61-65}$, we noticed that most of the genes upregulated in periodontitis were found to be expressed in plasma and myeloid cells. By contrast, genes downregulated in periodontitis were expressed in epithelial cells (Figure 6A). Using microarrays or bulk RNA sequencing reflected an average of expression profiles of all cells on whole biopsies, largely confounded by the enormous change in cell proportion; thus, differential expression analysis within individual cell types was performed (Figure 6B). In endothelial cells, HLA class II molecules $H L A-D R B 5$ and $C L E C 3 B$ associated with extracellular proteolysis were upregulated in periodontitis. In fibroblast, a metalloprotease $A D A M 12$ and $C F B$ involved in activating B cells was increased in disease. The expression level of two $\mathrm{C}-\mathrm{C}$ motif chemokine ligands, $C C L 4 L 2$ and $C C L 3 L 3$, was elevated in myeloid from patients. We also found PDCD1 was highly upregulated in the CD4_CTLA4 cluster from periodontitis, which indicates that PD-1 pathway may contribute to the protective effect of Treg in disease stage ${ }^{66}$. CTSW related to cytotoxic capacity was found to be upregulated in CD8_GZMK in periodontitis. These genes, previously masked in the mean expression data, provided novel insights for the characterization of periodontitis and will be helpful in advancing its therapy.

Periodontitis is a process of inflammation in the gingival tissue, which involves the cross talk of multiple cell types. Here, we used CellPhoneDB ${ }^{67}$ to profile the communication among cell types in healthy and periodontitis tissues (Figure 6C). In both healthy and periodontitis tissues, the interactions between epithelial, endothelial, and fibroblast cells are the strongest, while others are relatively weak. Generally, the overall cell-cell interaction increased in the periodontitis tissue. We showed the top 10 cell-cell interactions increased in patients (Figure 6D). Myeloid and B cells interact more frequently with other cells. Further, significant differences in the relative contribution of chemokine receptor-ligand pairs could be observed

302 between healthy and diseased gingiva (Figure 6E). Notably, the expression of CCR5 ligand 
303 increased in periodontitis, which was consistent with our above findings. These pairs could play

304 important roles in inflammation by recruiting inflammatory associated cells.

\section{Discussion}

307 Here, we present the first transcriptomic profiling of a total of 29,967 single cells of human 308 gingival tissue using the scRNA-seq method. By identifying a comprehensive catalog of cell 309 types and their phenotypes, revealing the altered gene expression profiling and cell-cell 310 communication under diseased condition, our data highlight key areas for advances in the 311 biology of periodontitis that will be helpful in the diagnosis and treatment of periodontitis.

312 Firstly, the epithelial is more heterogeneous than hitherto appreciated. While previous 313 studies had indicated that a compromised epithelial barrier defense, enabling easier penetration

314 of toxins or bacteria into the connective tissue, constituted an important mechanism for 315 periodontitis, our data further identified specific cell clusters with decreased expression of 316 epithelial homeostasis-associated genes, that contributed to pathogenicity of periodontal disease.

317 Despite of the considerable efforts of previous researchers, molecular regulations of gingival 318 development remained elusive. Using single-cell sequencing, different TFs underlying the 319 regulation of individual epithelial phenotype were identified, including some novel molecules 320 which had never been reported in this field before. For example, $P O U 2 F 2$ was highly expressed 321 in SPN2. The role of $P O U 2 F 2$ in B cell and epithelial-derived cancer cells has been elucidated, 322 but studies related to gingival development are still lacking.

323 Moreover, we detected 3 types of endothelial cells, 2 types of fibroblasts and different types 324 of immune cells. We show the presence of the recently identified antigen-presenting endothelial 325 cells in periodontitis ${ }^{47}$, as revealed by the combined expression of CD31 and MHC class II 326 genes. We identified a fibroblast subset potentially involved in bone remodeling. Heterogeneity 327 of myeloid cells was depicted, and novel phenotypes of macrophage, hitherto considered 328 dichotomous, were revealed. Our analysis confirmed that the in vitro characterized M1 and M2 329 cells do not reproduce the given tissue featured with a distinct local environment ${ }^{68}$. More 330 samples are needed to study the function of different DC subgroups in periodontal and the 331 differentiation of macrophages under different disease states. CCR5-ligand upregulated in 332 cytotoxic CD8 T cells, might imply a potential treatment option by blocking cell recruitment.

333 Besides, some genes associated with pro-inflammatory reactions were found to be expressed in 
334 the clinically healthy individuals, suggesting the complicated mechanisms involved in the oral

335 immune system to maintain immunological homeostasis.

336 Finally, we further investigated the biology of periodontitis in three directions: comparison

337 between healthy and periodontitis group; profiles of known periodontitis related genes; and the

338 cell-cell communication alteration between conditions. Our single-cell profiles not only provide

339 an abundance of resources on cell types and interaction networks in the human gingiva, but also

340 offer insights into the biological foundation of periodontal homeostasis and diseases, which

341 potentially serve as the basis of therapeutic options.

342 In gingiva, an important soft tissue within the periodontium, the number of epithelial cells is

343 far more than the number of immune cells. Under pathological conditions, cell types are not

344 affected equally in the development of periodontitis. Thus, scRNA-seq becomes the most

345 impartial and effective approach to obtain the transcriptome of each cell type in the gingiva.

346 Admittedly, the complexity of the periodontitis could not be fully grasped, as the gingiva was

347 located in restricted areas and the number of samples that were sequenced was also limited.

348 Nevertheless, given the robustness of scRNA-seq, it is possible to scale up the current study to

349 provide much improved resolution in the future.

351 Material and Methods

\section{Sample collection and ethics approval}

353 Collection of samples was approved by the Ethics Committee of Shanghai 9th People's Hospital

354 in China (SH9H-2019-T158-2). The experiments conformed to the principles of the Helsinki

355 Declaration revised in 2008. All donors gave informed consent prior to participation into the

356 study. Clinical assessment and biopsy sampling were conducted at the Department of Oral

357 Implantology, Shanghai 9th People's Hospital, Shanghai Jiaotong University, China. For

358 scRNA-seq, periodontal tissues of two healthy individuals and two patients with periodontitis

359 were collected. For inclusion in this study, patients with Stage III or IV periodontitis were

360 diagnosed according to the new classification of periodontitis ${ }^{69}$. Samples were collected from

361 healthy volunteers during crown lengthening procedure and from patients with periodontitis

362 during open flap debridement procedure. The demographic information and clinical parameters

363 of the two groups are shown in Supplementary Table 1. 


\section{Immunofluorescence}

366 Human periodontal soft tissue biopsies were incubated with primary antibodies diluted in 3\%

$367 \mathrm{BSA} / \mathrm{PBS}$ overnight at $4^{\circ} \mathrm{C}$. Primary antibodies used include Decorin (1:50, ab175404, Abcam),

368 Osteoglycin (1:50, sc-374463, Santa Cruz), HLA-DR (1:50, ab92511, Abcam), and CD31 (1:50, 369 ab9498, Abcam). Next day samples were incubated in Alexa-fluor 488, 594 Goat anti-Mouse or 370 Goat anti-Rabbit secondary antibodies (Jackson Immunoresearch). Nuclei were counterstained 371 with DAPI. Pictures were acquired using Zeiss LSM 880.

\section{Preparation of single-cell suspensions}

374 Once the sample was retrieved, it was dissociated and processed for scRNA-seq immediately.

375 Periodontal soft tissue samples were minced into small fragments of less than $1 \mathrm{~mm} 3$ by surgical 376 scissors and dissociated into single cells in dissociation solution ( $2 \mathrm{mg} / \mathrm{mL}$ IV collagenase, 2 377 Unit/mL Dispase II in $\mathrm{Ca}_{2}{ }^{+}$- and $\mathrm{Mg}^{+}$-free HBSS) covered with tinfoil on a shaker (shaking 378 speed of $200 \mathrm{rpm}$ ) at $37^{\circ} \mathrm{C}$ for $60 \mathrm{~min} .0 .1 \mu \mathrm{g} / \mathrm{mL}$ DNase I was added in the last $10 \mathrm{~min}$. The 379 dissociated tissue was filtered to ensure single-cell suspension using $100 \mu \mathrm{m}, 40 \mu \mathrm{m}$ cell strainers 380 (Falcon) successively. Cells were subjected to red blood cell lysis for $10 \mathrm{~min}$ and centrifuged and 381 resuspended $(500 \mathrm{~g}, 10 \mathrm{~min})$ twice. After resuspension in defined volumes of PBS $+0.4 \% \mathrm{BSA}$, $38210 \mu \mathrm{L}$ of the cell suspension was used for cell counting by an automated cell counter 383 (Thermofisher) to determine the concentration of live cells. Single-cell samples with final cell 384 viability above $90 \%$ and final concentration of $600-1200$ cells $\mu \mathrm{L}^{-1}$ were stored on ice until 385 further processing. The whole procedure was performed on ice whenever possible.

\section{Single-cell RNA sequencing and read processing}

388 Single cells from independent periodontal samples were captured in four batches using the 10X

389 Chromium system (10X Genomics). The cells were partitioned into Gel Bead-In-Emulsions and 390 barcoded cDNA libraries, then prepared using the Chromium Single Cell 3' library \& Gel Bead 391 Kit v3 (10X Genomics). Single-cell libraries were sequenced in 100 bp paired-end configuration 392 using an Illumina NovaSeq and mapped to the GRCh38 human reference genome using the Cell 393 Ranger toolkit (version 3.0.0). The preliminary data analysis generated a file containing a 394 barcodes table, a genes table, and a gene expression matrix. Next, we obtained an overview 
website containing a considerable amount of information, such as number of cells, median number of detected genes, sequencing saturation, and sequencing depth.

\section{Filtering and normalization of scRNA-seq data}

399 We installed R (version 3.5.1) and Seurat R package (version 3.1.5) for downstream analysis.

400 First, the substantial background levels of ambient RNA in the single-cell suspension caused 401 problems for subsequent analysis. Thus, we applied SoupX (version 1.4.5) for background 402 correction. Next, for quality control of each matrix, lowly detected genes $(<0.1 \%$ cells $)$ and cells 403 with a small number of genes (<350 genes) were discarded from the downstream analysis. Then, 404 we filtered out unhealthy cells that generally have high mitochondrial mRNA loads (>20\%) and 405 high ribosome RNA loads (>40\%). We found that different cell types expressed different 406 numbers of genes, particularly between immune and non-immune cells. Thus, we applied a 407 slightly different criteria to remove supposed ambient RNA contamination (<1700 UMI for 408 healthy detected per cell) and potential double droplets ( $>6500$ genes for healthy, and $>4000$ 409 genes for periodontitis tissues detected per cell). After the step above, we obtained 10,501 high 410 quality periodontitis cells (median UMI: 3544; median 1203 genes/cell) and 19,977 healthy 411 periodontal cells (median UMI: 16,455; median 3169 genes/cell).

\section{Dimension reduction, unsupervised clustering, annotation, and visualization}

414 The expression value of each gene was first normalized by TPM/10 and then log-transformed 415 (NormalizeData function in Seurat with default parameters). Using the variation stabilizing 416 transformation (vst) method, the top 2000 variable genes were selected in each matrix and were 417 used as input for the 'FindIntegrationAnchors' function. The four expression matrices were then 418 integrated with the 'IntegrateData' function. The integrated data were dimension reduced with 419 principal component analysis (PCA; top 30 dimensions) first and then further reduced to two 420 dimensions with UMAP which was also used to visualize the clusters. The nearest neighbors 421 were defined among cells with KNN method (FindNeighbors), and cells were then grouped with 422 Louvain algorithm (FindClusters in Seurat, resolution equal to 1.5; PCA:top 25 dimensions). 423 Specifically, we removed one cluster, considered as contamination, due to the co-expressed 424 markers of plasma and epithelial cells (data not shown). Finally, we retained a total of 29,967 425 cells after stringent quality controls for further analysis, with 19,806 (66.09\%) from healthy 
426 tissues and 10,161 from the periodontitis tissues. Annotation of the clusters was performed by

427 checking known markers for cell types that potentially would exist in the sample, and some

428 clusters were merged as they were annotated as a major cell type. Average expression levels of

429 each subtype marker was calculated by AddModuleScore in Seurat with default parameters. For

430 sub-clustering, cells from a major cell type were taken as the input. We performed dimension

431 reduction, clustering, and annotation using the same method as described above.

432

\section{Detection of differentially expressed genes (DEGs)}

434 To obtain the DEG list of each cluster, only the genes expressed in more than $30 \%$ of that cluster

435 were considered, and the expression in all other cells was used as background. For statistical test, 436 we used the default Wilcoxon test implemented in Seurat. DEGs were defined as genes whose

437 log fold-change was over 0.2 compared to the background, and with a q-value (FDR) smaller 438 than 0.05 .

Gene set enrichment analysis

441 We conducted the gene set enrichment analysis for DEGs of each cluster using clusterProfiler ${ }^{70}$,

442 GSVA, and GSEABase packages, with which the enriched GO biological process terms were 443 calculated.

\section{SCENIC analysis}

446 SCENIC analysis was conducted as described previously. ${ }^{36} \mathrm{We}$ used the pySCENIC package

447 (version 0.10.3), a lightning-fast python implementation of the SCENIC pipeline. The

448 differentially activated TFs of each subcluster were identified by the Wilcoxon rank sum test

449 against all the other cells of the same cell type.

\section{Reconstructing gingival epithelial differentiation trajectories using Monocle2}

452 Cell fate decisions and pseudotime trajectories were reconstructed using the Monocle $2 \mathrm{R}$

453 package (version 2.10.1). Firstly, these Seurat data which included eight types of epithelial were

454 imported into Monocle2. Genes that were expressed in at least ten cells were used, and only

455 genes expressed in .3\% of cells were kept. We used thresholds on the cells local density (r) and

456 nearest distance $(\mathrm{d})$ to determine the number of clusters. We used 'dpFeature' to find the 
differential genes (mean_expression $>=0.3 \&$ dispersion_empirical $>=1 *$ dispersion_fit). These

458 DEGs, which were a set of ordering genes, were then used to perform the dimension reduction 459 and the trajectory analysis. Once we established a trajectory, we used the differential Gene Test

460 function to find genes that had an expression pattern that varied according to pseudotime.

\section{RNA velocity analysis}

463 RNA velocity analysis was performed using the scVelo (version 0.2.1). Briefly,

464 spliced/unspliced reads were annotated by velocyto.py with CellRanger (version 3.0.0),

465 generating BAM files and then saved in .loom files. The .loom files were then loaded to python

466 (version 3.8.3) using the anndata.read_loom function to generate count tables for splicing and

467 unsplicing reads. Then we used dynamical model from scVelo to calculate. Lastly, the velocity

468 vector arrows were projected onto the UMAP plot which was obtained in Seurat.

\section{Profiling the cell-cell communication in healthy and periodontitis samples}

The cell-cell communication was measured by quantification of ligand-receptor pairs among

472 different cell types. Gene expression matrices and metadata with major cell annotations were

473 used as input for the CellphoneDB or CellChat ${ }^{71}$ software. The default CellPhoneDB database

474 and parameters were used. Healthy and periodontitis data was computed separately. The cell-cell

475 network was visualized with circlize (Version 0.4.10).

\section{Author contributions}

478 H.L. and B.L. conceived the project. S.Q., Q.H., Y.Z., and L.Z. participated in the

479 data analysis. J.S. advised the data analysis. S.Q., L.Z, and J.S. collected the human donor

480 gingiva, performed the phenotyping and dissection. Q.H. and L.Z. prepared the nuclei sample

481 and performed the single-nuclei RNA-seq. S.Q., Q.H., H.L. and B.L. wrote the manuscript with

482 input from all other authors. All authors proofread the manuscript.

\section{Competing interests}

485 The authors declare no competing interests. 


\section{References}

4881 Nunez, J., Vignoletti, F., Caffesse, R. G. \& Sanz, M. Cellular therapy in periodontal 489 regeneration. Periodontol 2000 79, 107-116, doi:10.1111/prd.12250 (2019).

4902 Ouchi, T. \& Nakagawa, T. Mesenchymal stem cell-based tissue regeneration therapies 491 for periodontitis. Regen Ther 14, 72-78, doi:10.1016/j.reth.2019.12.011 (2020).

4923 Tonetti, M. S., Van Dyke, T. E. \& working group 1 of the joint, E. F. P. A. A. P. w. 493 Periodontitis and atherosclerotic cardiovascular disease: consensus report of the Joint EFP/AAPWorkshop on Periodontitis and Systemic Diseases. J Periodontol 84 Suppl 4S, S24-S29, doi:10.1902/jop.2013.1340019 (2013).

4964 Genco, R. J. \& Sanz, M. Clinical and public health implications of periodontal and systemic diseases: An overview. Periodontology 2000 83, 7-13, doi:10.1111/prd.12344 (2020).

4995 Global, regional, and national incidence, prevalence, and years lived with disability for 301 acute and chronic diseases and injuries in 188 countries, 1990-2013: a systematic analysis for the Global Burden of Disease Study 2013. Lancet 386, 743-800, doi:10.1016/s0140-6736(15)60692-4 (2015). Bartold, P. M. \& Van Dyke, T. E. Periodontitis: a host-mediated disruption of microbial homeostasis. Unlearning learned concepts. Periodontol 2000 62, 203-217, doi:10.1111/j.1600-0757.2012.00450.x (2013).

5098 Donos, N. The periodontal pocket. Periodontol 2000 76, 7-15, doi:10.1111/prd.12203

510 (2018).

5119 Nunez, J., Vignoletti, F., Caffesse, R. G. \& Sanz, M. Cellular therapy in periodontal 512 regeneration. Periodontology 2000 79, 107-116, doi:10.1111/prd.12250 (2019).

51310 Moutsopoulos, N. M. \& Konkel, J. E. Tissue-Specific Immunity at the Oral Mucosal 514 Barrier. Trends Immunol 39, 276-287, doi:10.1016/j.it.2017.08.005 (2018).

51511 Parisi, L. et al. Macrophage Polarization in Chronic Inflammatory Diseases: Killers or Builders? J Immunol Res 2018, 8917804, doi:10.1155/2018/8917804 (2018). 
51712 Smith, P. C., Martínez, C., Martínez, J. \& Mcculloch, C. A. Role of Fibroblast

518 Populations in Periodontal Wound Healing and Tissue Remodeling. Front. Physiol. 10, 519 270- (2019).

52013 Alvarez, C. et al. Regulatory T Lymphocytes in Periodontitis: A Translational View.

$521 \quad$ Mediators Inflamm. 2018, 7806912, doi:10.1155/2018/7806912 (2018).

52214 Glowacki, A. J. et al. Prevention of inflammation-mediated bone loss in murine and 523 canine periodontal disease via recruitment of regulatory lymphocytes. Proc. Natl. Acad.

524 Sci. U. S. A. 110, 18525-18530, doi:10.1073/pnas.1302829110 (2013).

52515 Sima, C., Viniegra, A. \& Glogauer, M. Macrophage immunomodulation in chronic $526 \quad$ osteolytic diseases - the case of periodontitis. Journal of Leukocyte Biology 105, 473527 487, doi:10.1002/jlb.1ru0818-310r (2019).

52816 Xie, Y., Sun, M., Xia, Y. \& Shu, R. An RNA-seq screen of P. gingivalis LPS treated 529 human gingival fibroblasts. Arch Oral Biol 88, 77-84, 530 doi:10.1016/j.archoralbio.2018.01.002 (2018).

53117 Wang, P. L., Azuma, Y., Shinohara, M. \& Ohura, K. Toll-like receptor 4-mediated signal 532 pathway induced by Porphyromonas gingivalis lipopolysaccharide in human gingival fibroblasts. Biochem Bioph Res Co 273, 1161-1167, doi:10.1006/bbrc.2000.3060 (2000). Wu, X., Zhang, G., Feng, X., Li, P. \& Tan, Y. Transcriptome analysis of human periodontal ligament fibroblasts exposed to Porphyromonas gingivalis LPS. Arch Oral Biol 110, 104632, doi:10.1016/j.archoralbio.2019.104632 (2020).

20 Kang, W. et al. Time-Course Transcriptome Analysis for Drug Repositioning in Ahn, S. H. et al. Transcriptome profiling analysis of senescent gingival fibroblasts in response to Fusobacterium nucleatum infection. PLoS One 12, e0188755, Fusobacterium nucleatum-Infected Human Gingival Fibroblasts. Front Cell Dev Biol 7, 204, doi:10.3389/fcell.2019.00204 (2019).

21 Kang, W., Sun, T., Tang, D., Zhou, J. \& Feng, Q. Time-Course Transcriptome Analysis of Gingiva-Derived Mesenchymal Stem Cells Reveals That Fusobacterium nucleatum Triggers Oncogene Expression in the Process of Cell Differentiation. Front Cell Dev Biol 7, 359, doi:10.3389/fcell.2019.00359 (2019). 
54722 Demmer, R. T. et al. Transcriptomes in Healthy and Diseased Gingival Tissues. Journal 548 of Periodontology 79, 2112-2124, doi:10.1902/jop.2008.080139 (2008).

54923 Kim, Y. G. et al. Transcriptome sequencing of gingival biopsies from chronic

550 periodontitis patients reveals novel gene expression and splicing patterns. Hum Genomics 551 10, 28, doi:10.1186/s40246-016-0084-0 (2016).

55224 Horie, M. et al. Transcriptome analysis of periodontitis-associated fibroblasts by CAGE 553 sequencing identified DLX5 and RUNX2 long variant as novel regulators involved in $554 \quad$ periodontitis. Sci Rep 6, 33666, doi:10.1038/srep33666 (2016).

55525 Lundmark, A. et al. Gene expression profiling of periodontitis-affected gingival tissue by $556 \quad$ spatial transcriptomics. Sci Rep 8, 9370, doi:10.1038/s41598-018-27627-3 (2018).

55726 Young, M. D. \& Behjati, S. SoupX removes ambient RNA contamination from droplet

27 Butler, A., Hoffman, P., Smibert, P., Papalexi, E. \& Satija, R. Integrating single-cell transcriptomic data across different conditions, technologies, and species. Nat Biotechnol 36, 411-420, doi:10.1038/nbt.4096 (2018).

Hart, T. C. et al. A mutation in the SOS1 gene causes hereditary gingival fibromatosis type 1. Am J Hum Genet 70, 943-954, doi:10.1086/339689 (2002). Bayram, Y. et al. REST Final-Exon-Truncating Mutations Cause Hereditary Gingival Fibromatosis. Am J Hum Genet 101, 149-156, doi:10.1016/j.ajhg.2017.06.006 (2017). Dabija-Wolter, G., Bakken, V., Cimpan, M. R., Johannessen, A. C. \& Costea, D. E. In vitro reconstruction of human junctional and sulcular epithelium. J Oral Pathol Med 42, 396-404, doi:10.1111/jop.12005 (2013).

57232 Lee, J. Y. et al. Serum Amyloid A Proteins Induce Pathogenic Th17 Cells and Promote 573 Inflammatory Disease. Cell 180, 79-91 e16, doi:10.1016/j.cell.2019.11.026 (2020).

57433 Singhal, S. S. et al. Antioxidant role of glutathione S-transferases: 4-Hydroxynonenal, a $575 \quad$ key molecule in stress-mediated signaling. Toxicol Appl Pharmacol 289, 361-370, doi:10.1016/j.taap.2015.10.006 (2015). 
$57734 \quad$ Blanke, K. L. et al. Polymorphisms in the carcinogen detoxification genes CYB5A and 578 CYB5R3 and breast cancer risk in African American women. Cancer Causes Control 25, 1513-1521, doi:10.1007/s10552-014-0454-7 (2014).

$58035 \quad$ Hanzelmann, S., Castelo, R. \& Guinney, J. GSVA: gene set variation analysis for microarray and RNA-seq data. BMC Bioinformatics 14, 7, doi:10.1186/1471-2105-14-7 (2013).

36 Aibar, S. et al. SCENIC: single-cell regulatory network inference and clustering. Nat Methods 14, 1083-1086, doi:10.1038/nmeth.4463 (2017). $\mathrm{Xu}$, C. et al. Epithelium-specific Runx2 Knockout Mice Display Junctional Epithelium and Alveolar Bone Defects. Oral Dis, doi:10.1111/odi.13647 (2020).

38 Lee, H. K. et al. The odontogenic ameloblast-associated protein (ODAM) cooperates with RUNX2 and modulates enamel mineralization via regulation of MMP-20. J Cell Biochem 111, 755-767, doi:10.1002/jcb.22766 (2010).

39 Kawasaki, K. et al. Expression of Sox genes in tooth development. Int J Dev Biol 59,

$59240 \quad$ Trapnell, C. et al. The dynamics and regulators of cell fate decisions are revealed by pseudotemporal ordering of single cells. Nat Biotechnol 32, 381-386, doi:10.1038/nbt.2859 (2014).

Qiu, X. et al. Reversed graph embedding resolves complex single-cell trajectories. Nat Methods 14, 979-982, doi:10.1038/nmeth.4402 (2017). characteristics of human gingival junctional epithelium. BMC Oral Health 14, 30,

$60043 \quad$ Nakamura, M. Histological and immunological characteristics of the junctional epithelium. Jpn Dent Sci Rev 54, 59-65, doi:10.1016/j.jdsr.2017.11.004 (2018).

60244 Chai, J. et al. CCN1 induces a reversible epithelial-mesenchymal transition in gastric 603 epithelial cells. Lab Invest 90, 1140-1151, doi:10.1038/labinvest.2010.101 (2010).

60445 Sun, B. K. et al. CALML5 is a ZNF750- and TINCR-induced protein that binds stratifin 605 to regulate epidermal differentiation. Genes Dev 29, 2225-2230, doi:10.1101/gad.267708.115 (2015). 
60746 Fujimoto, N. et al. Single-cell mapping reveals new markers and functions of lymphatic

608 endothelial cells in lymph nodes. PLoS Biol 18, e3000704,

609 doi:10.1371/journal.pbio.3000704 (2020).

61047 Han, X. et al. Construction of a human cell landscape at single-cell level. Nature 581, 611 303-309, doi:10.1038/s41586-020-2157-4 (2020).

61248 Hinz, B. Matrix mechanics and regulation of the fibroblast phenotype. Periodontology

6132000 63, 14-28, doi:10.1111/prd.12030 (2013).

61449 Armas-Gonzalez, E. et al. Role of CXCL13 and CCL20 in the recruitment of B cells to 615 inflammatory foci in chronic arthritis. Arthritis Res Ther 20, 114, doi:10.1186/s13075616 018-1611-2(2018).

61750 Kim, S. H., Han, S. Y., Azam, T., Yoon, D. Y. \& Dinarello, C. A. Interleukin-32: a

618 cytokine and inducer of TNFalpha. Immunity 22, 131-142,

619 doi:10.1016/j.immuni.2004.12.003 (2005).

62051 Chen, X. et al. Effects of Osteoglycin (OGN) on treating senile osteoporosis by

621 regulating MSCs. BMC Musculoskelet Disord 18, 423, doi:10.1186/s12891-017-1779-7

622 (2017).

62352 Rucci, N. et al. The glycosaminoglycan-binding domain of PRELP acts as a cell type-

624 specific NF-kappaB inhibitor that impairs osteoclastogenesis. J Cell Biol 187, 669-683, 625 doi:10.1083/jcb.200906014 (2009).

62653 Zhang, L. et al. Single-Cell Analyses Inform Mechanisms of Myeloid-Targeted

627 Therapies in Colon Cancer. Cell 181, 442-459 e429, doi:10.1016/j.cell.2020.03.048

628 (2020).

62954 Tang-Huau, T. L. et al. Human in vivo-generated monocyte-derived dendritic cells and 630 macrophages cross-present antigens through a vacuolar pathway. Nat Commun 9, 2570, 631 doi:10.1038/s41467-018-04985-0 (2018).

63255 Jahrsdorfer, B. et al. Granzyme B produced by human plasmacytoid dendritic cells 633 suppresses T-cell expansion. Blood 115, 1156-1165, doi:10.1182/blood-2009-07-235382 634 (2010).

63556 Shang, Y. et al. The transcriptional repressor Hes1 attenuates inflammation by regulating 636 transcription elongation. Nature Immunology (2016). 
63757 Bergen, V., Lange, M., Peidli, S., Wolf, F. A. \& Theis, F. J. Generalizing RNA velocity

638 to transient cell states through dynamical modeling. Nat Biotechnol, doi:10.1038/s41587-

639 020-0591-3 (2020).

64058 Murray, P. J. et al. Macrophage activation and polarization: nomenclature and

641 experimental guidelines. Immunity 41, 14-20, doi:10.1016/j.immuni.2014.06.008 (2014).

64259 Dapunt, U., Maurer, S., Giese, T., Gaida, M. M. \& Hansch, G. M. The macrophage

643 inflammatory proteins MIP1alpha (CCL3) and MIP2alpha (CXCL2) in implant-

644 associated osteomyelitis: linking inflammation to bone degradation. Mediators Inflamm

2014, 728619, doi:10.1155/2014/728619 (2014).

64660 van den Brink, S. C. et al. Single-cell sequencing reveals dissociation-induced gene

647 expression in tissue subpopulations. Nat Methods 14, 935-936, doi:10.1038/nmeth.4437

648 (2017).

64961 Davanian, H. et al. Gene expression profiles in paired gingival biopsies from

650 periodontitis-affected and healthy tissues revealed by massively parallel sequencing.

$651 \quad$ PLoS One 7, e46440, doi:10.1371/journal.pone.0046440 (2012).

65262 Demmer, R. T. et al. Transcriptomes in healthy and diseased gingival tissues. $J$

$653 \quad$ Periodontol 79, 2112-2124, doi:10.1902/jop.2008.080139 (2008).

65463 Beikler, T., Peters, U., Prior, K., Eisenacher, M. \& Flemmig, T. F. Gene expression in

655 periodontal tissues following treatment. BMC Med Genomics 1, 30, doi:10.1186/1755-

656 8794-1-30 (2008).

65764 Becker, S. T. et al. Peri-implantitis versus periodontitis: functional differences indicated

658 by transcriptome profiling. Clin Implant Dent Relat Res 16, 401-411,

659 doi:10.1111/cid.12001 (2014).

66065 Lundmark, A. et al. Transcriptome analysis reveals mucin 4 to be highly associated with

661 periodontitis and identifies pleckstrin as a link to systemic diseases. Sci Rep 5, 18475, 662 doi:10.1038/srep18475 (2015).

66366 Wafula, P. O. et al. PD-1 but not CTLA-4 blockage abrogates the protective effect of 664 regulatory T cells in a pregnancy murine model. Am J Reprod Immunol 62, 283-292, 665 doi:10.1111/j.1600-0897.2009.00737.x (2009). 
66667 Efremova, M., Vento-Tormo, M., Teichmann, S. A. \& Vento-Tormo, R. CellPhoneDB:

667 inferring cell-cell communication from combined expression of multi-subunit ligand-

668 receptor complexes. Nat Protoc 15, 1484-1506, doi:10.1038/s41596-020-0292-x (2020).

66968 Amit, I., Winter, D. R. \& Jung, S. The role of the local environment and epigenetics in

670 shaping macrophage identity and their effect on tissue homeostasis. Nature Immunology

$671 \quad$ 17, 18-25, doi:10.1038/ni.3325 (2016).

67269 Tonetti, M. S., Greenwell, H. \& Kornman, K. S. Staging and grading of periodontitis:

673 Framework and proposal of a new classification and case definition. J Clin Periodontol

$674 \quad 45$ Suppl 20, S149-S161, doi:10.1111/jcpe.12945 (2018).

67570 Yu, G., Wang, L. G., Han, Y. \& He, Q. Y. clusterProfiler: an R package for comparing

676 biological themes among gene clusters. OMICS 16, 284-287, doi:10.1089/omi.2011.0118

677 (2012).

67871 Jin, S. et al. Inference and analysis of cell-cell communication using CellChat.

$679 \quad 2020.2007 .2021 .214387$, doi:10.1101/2020.07.21.214387 \%J bioRxiv (2020). 
681 Figure 1 Overview of the clustering and annotation of the single-cell RNA sequencing data 682 for gingival tissues.

683 A. Schematic depicting anatomical regions analyzed in this study (The graph was created with 684 BioRender.com).

685 B. tSNE of the 29,967 cells profiled here, with each cell color-coded for (left to right): the 686 corresponding patient and the associated cell type.

687 C. Well-known markers scores for annotation of gingival cell types visualized by violin plot.

688 D. Proportion of cell types from periodontitis and healthy conditions. (Nor1 and Nor2 are 689 healthy donors; P1 and P2 are periodontitis patients)

690 E. Dot plot depicting gene expression levels and percentage of cells expressing genes associated 691 with periodontal disease according to the OMIM database.

693 Figure 2 The heterogeneity and developmental trajectory of gingival epithelial.

694 A. Visualization of human gingival epithelial cells in disease and healthy conditions separately.

695 B. Markers for annotation of gingival epithelial cell types visualized by violin plot.

696 C. Immunohistochemical showed the outermost layer of the epithelium experienced severe 697 ruptures in patients.

698 D. UMAP plots of epithelial cells, color-coded for functional molecules scores.

699 E. Heatmap showing the activity of TFs in each epithelial cell subtype. The TF activity is scored $700 \quad$ using scaled AUCell.

701 F. Monocle2-generated pseudotime trajectory of epithelial types imported from Seurat data, 702 colored by cell-name designation (left) and colored in a gradient from dark to light yellow 703 (right).

704 G. Heatmap for clustering the 30 genes that vary as a function of pseudotime. The 30 genes 705 were divided into four clusters, representing the genes at the beginning stage, the transitory 706 stage, and the end stage of developmental trajectory, respectively. 
708 Figure 3 Three endothelial subclusters identified from the human gingival cells.

709 A. UMAP visualization of three endothelial subclusters from human gingival cells.

710 B. Violin plots showing the expression distribution of selected genes associated with functions

711 in the endothelial clusters.

712 C. Top gene ontology term enrichment for endothelial clusters.

713 D. HLA-DRA showed expression specificity in the endothelial Endo_1. The gene expression

714 levels are normalized and transformed as $\ln (\mathrm{CPM} / 10)$.

715 E. Immunofluorescent (IF) staining validation of endothelial subtypes. Red color showed the 716 signal of HLA-DR staining; green color showed the signal of CD-31 staining (endothelial 717 marker); and blue color showed DAPI staining.

719 Figure 4 Two fibroblast subclusters identified from the human gingival cells.

720 A. UMAP visualization of two fibroblast subclusters from human gingival cells.

721 B. Violin plots showing the expression distribution of selected genes associated with functions

722 in the endothelial clusters. The gene expression levels are normalized and transformed as $\mathrm{ln}$ $723(\mathrm{CPM} / 10)$.

724 C. Differences in pathway activities scored per cell by GSVA between fibro_1 and fibro_2.

725 Shown are $t$ values from a linear model, corrected for fibro_1.

726 D. Immunofluorescent (IF) staining validation of fibroblast subtypes. The IF was performed on 727 subepithelial region and connective tissue, respectively. Red color showed the signal of 728 Decorin staining (fibroblast marker); green color showed the signal of Osteoglycin (OGN) 729 staining; and blue color showed DAPI staining.

730 E. Application of scHCL analysis for non-immune cells. Each row represents one cell type in 731 scHCL. Each column represents a cell cluster in our dataset. Pearson correlation coefficient 732 was used to evaluate cell-type gene expression similarity. Red indicates a high correlation; 733 blue indicates a low correlation. 


\section{Figure 5 The heterogeneity of myeloid and $\mathbf{T}$ cells.}

736 A. UMAP visualization of seven myeloid clusters from human gingival cells.

737 B. Relative proportion of myeloid cell subsets from different origin (healthy $=2$ and

738 periodontitis $=2$ ).

739 C. Bubble heatmap showing marker genes across seven myeloid clusters from (A). Dot size

740 indicates fraction of expressing cells, colored according to z-score normalized expression 741 levels.

742 D. Violin plots showing the expression of angiogenesis- and phagocytosis-related genes in three 743 macrophages clusters. The gene expression levels are normalized and transformed as $\mathrm{ln}$ $744 \quad(\mathrm{CPM} / 10)$.

745 E. Velocity field projected onto the UMAP plot of three macrophages (arrows represent average 746 velocity).

747 F. UMAP visualization of seven myeloid clusters from human gingival cells.

748 G. Relative proportion of $\mathrm{T}$ cell subsets from different origin (healthy=2 and periodontitis=2).

749 H. Heatmap of T cell top 5 DEGs in each cell cluster in healthy and periodontitis.

Figure 6 The difference among gingival cell types in healthy and periodontitis conditions.

752 A. Bubble heatmap showing reported genes associated diseases clusters from (Figure 1B). Rose

753 stands for reported increase in periodontitis, and turquoise stands for reported decrease in

754 periodontitis. Dot size indicates fraction of expressing cells, colored according to z-score 755 normalized expression levels.

756 B. Violin plots showing the expression of gene specific increasing in different clusters of 757 periodontitis. The gene expression levels are normalized and transformed as $\ln (\mathrm{CPM} / 10)$.

758 C. Cell-cell interaction network in healthy and periodontitis condition. Colors and widths of 759 edges represent number of interaction pairs between cell types.

760 D. The top 10 cell-cell interactions with the most significant increase in patients than healthy.

761 The relative contribution of receptor-ligand in CCL pathway in normal and periodontitis.

762 Yellow indicates that the common receptor-ligand is present in both the patient and the 763 normal. 
A.
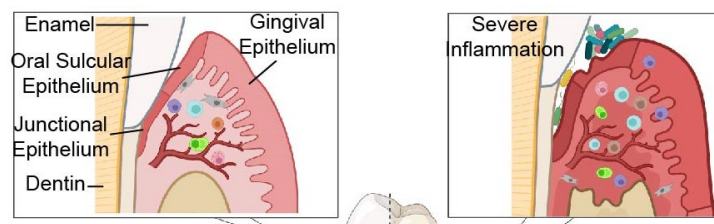

C.

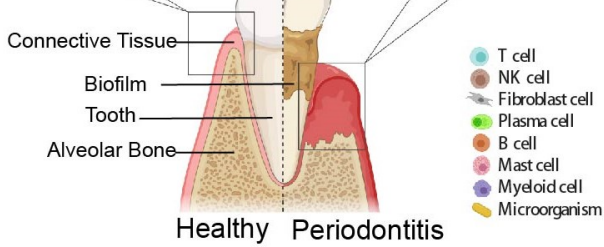

B.

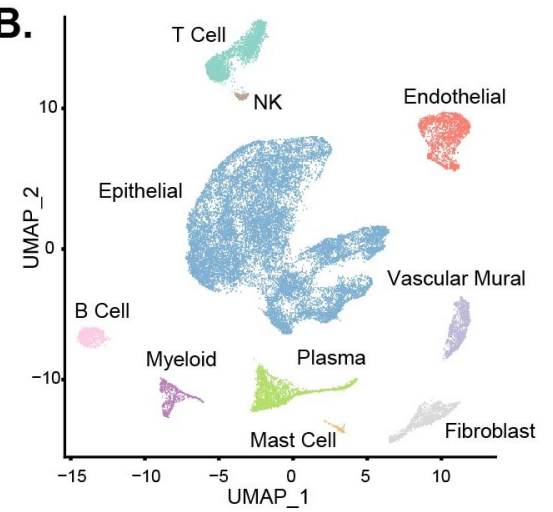

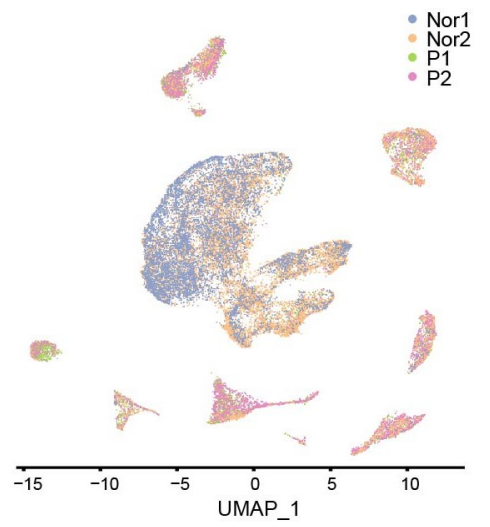

\section{KRT14, CDH1, EPCAM, KRT76, KRT16}
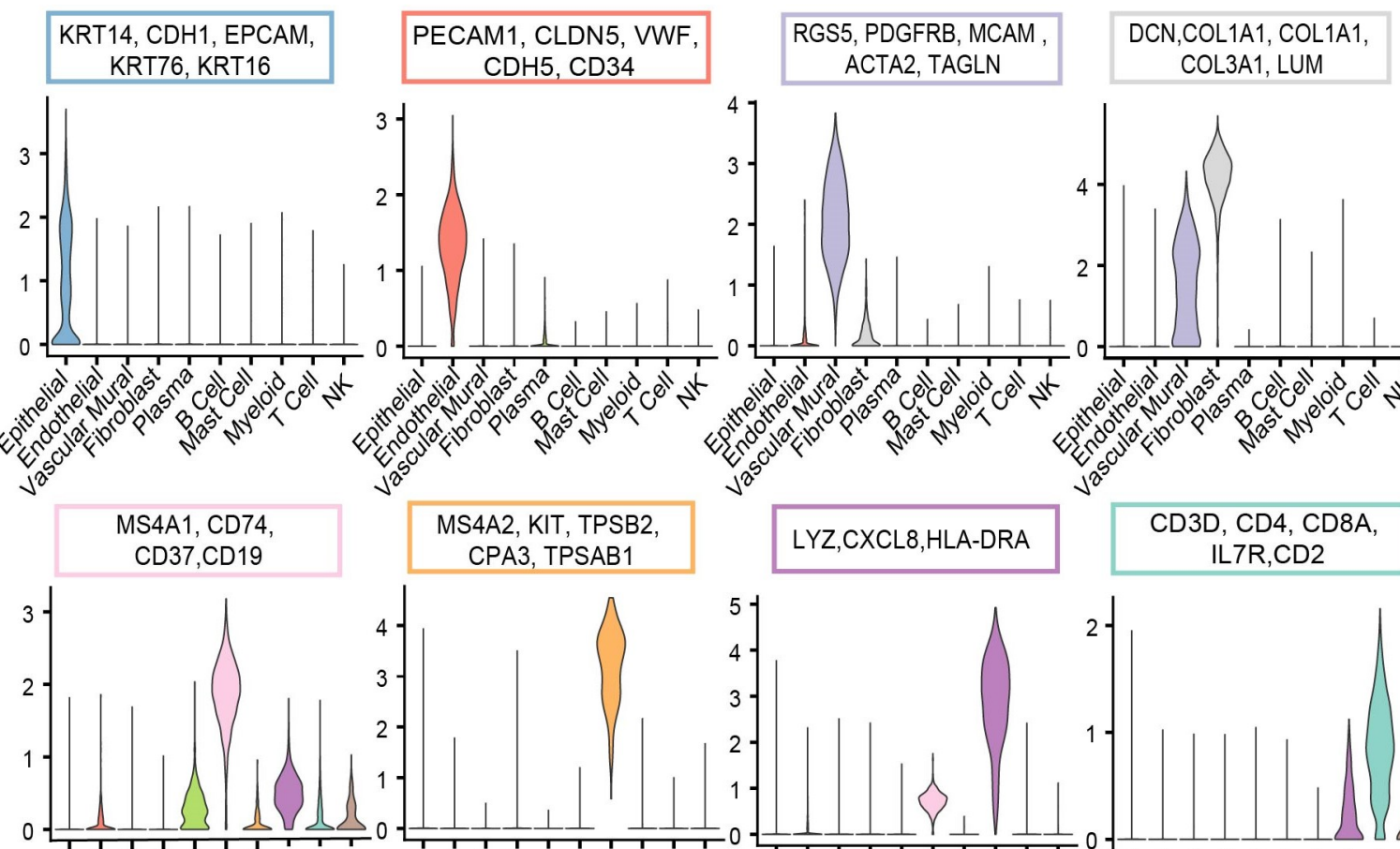

CD79A, IGLC2, IGHG1,
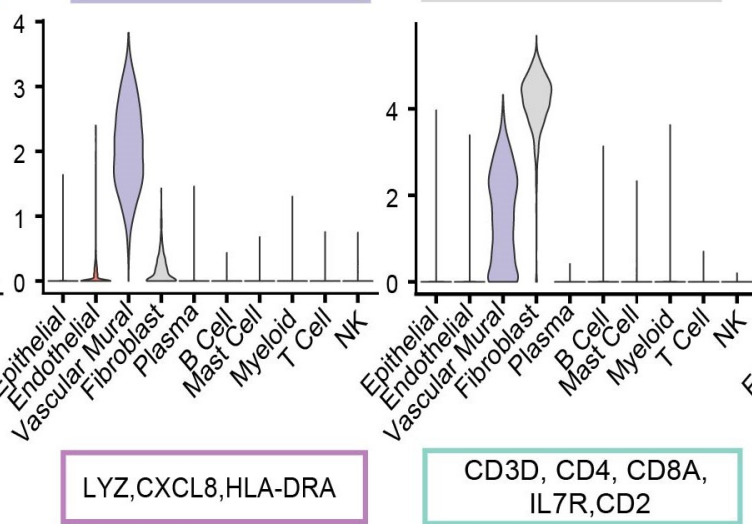
IGHG2, IGHG3
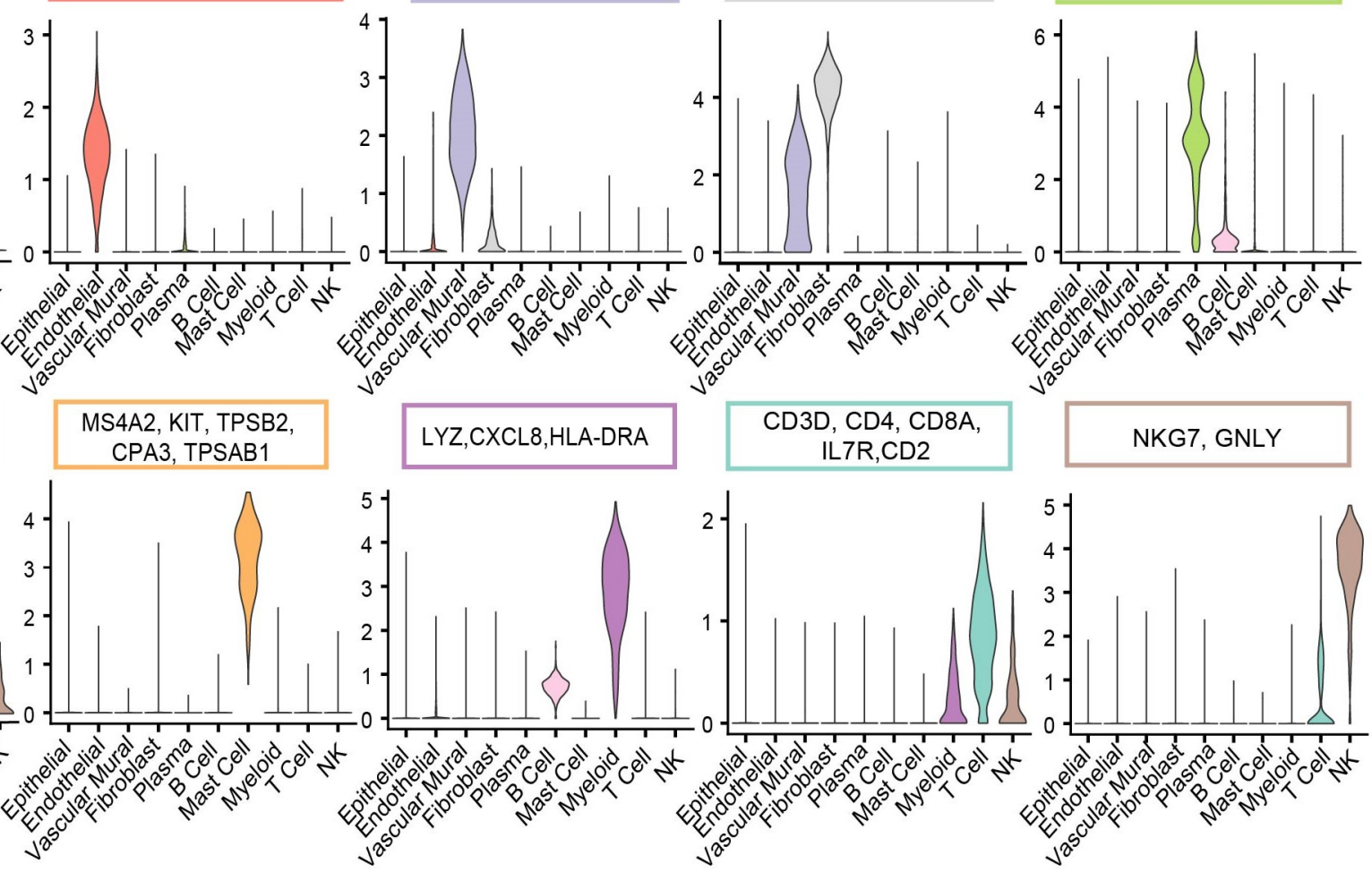

NKG7, GNLY

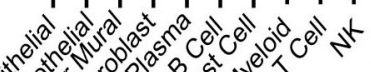

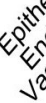

D.

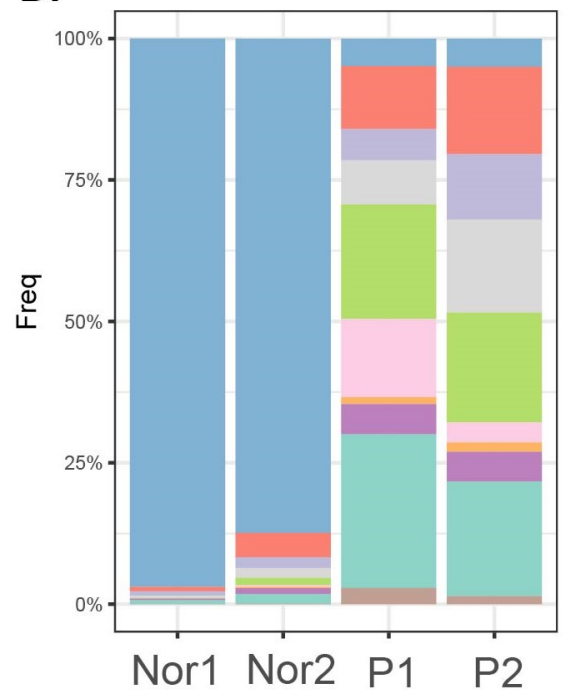

E.

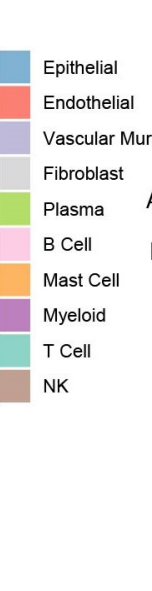

Percent Expressed 00 • $25 \bullet 50 \quad 75$ Average Expression

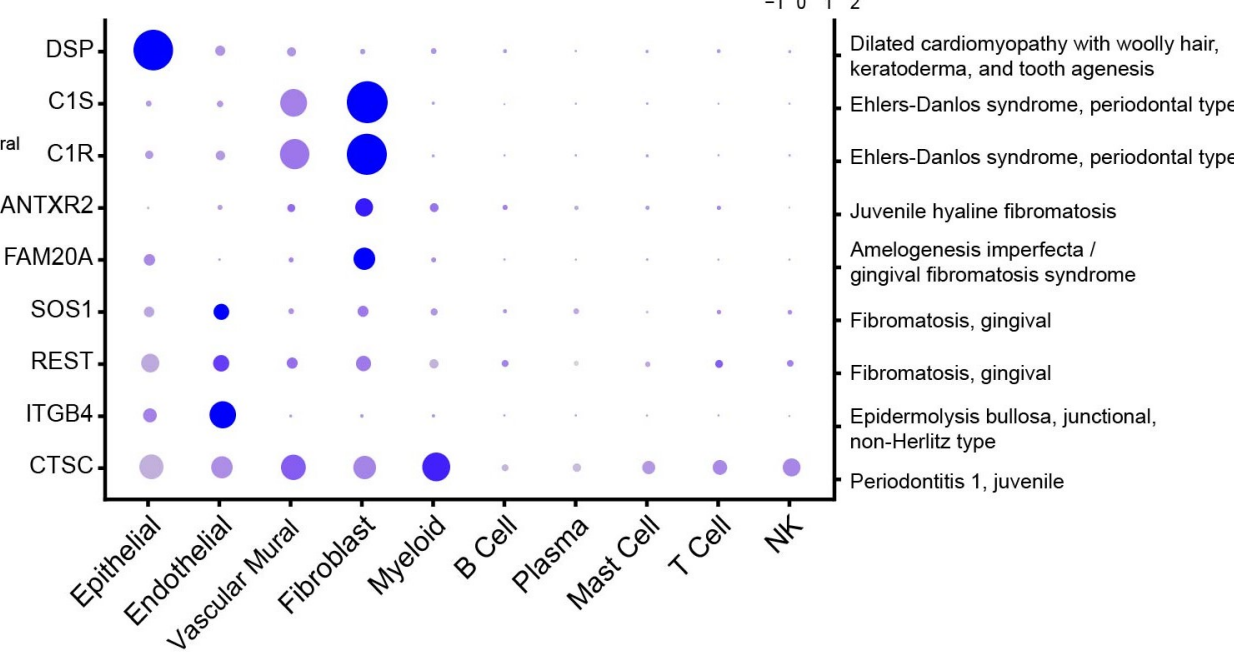


A.

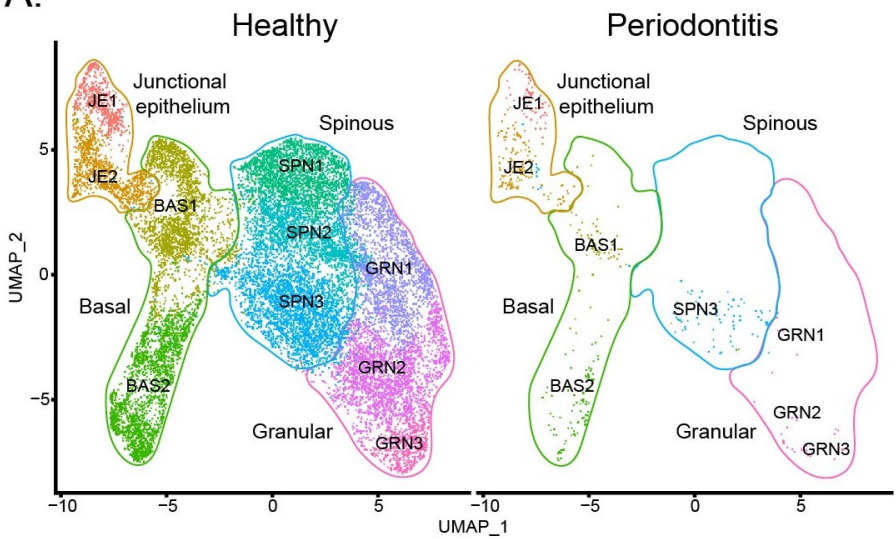

C.
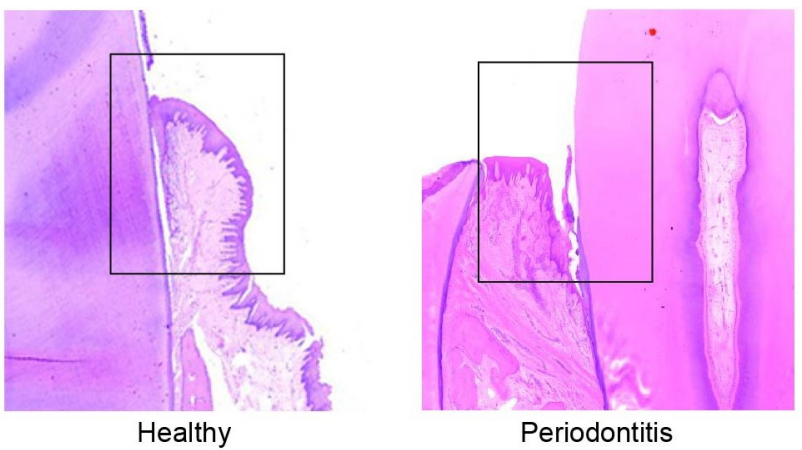

D. Amelogenesis Serum Amyloid A Family $\begin{array}{cc}\text { HLA-DRs } & \text { Mitosis } \\ \text { (HLA-DRB1,HLA-DRA) } & \text { (MKI67,UBE2C,TOP2A) }\end{array}$
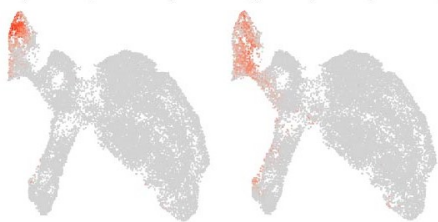

Detoxification Epidernal Differentiation Com (GSTA4,CYB5A) (SPRR3,,S100A7,CRNN)

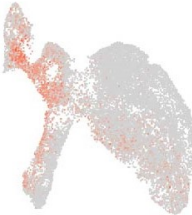
(SLPI,SPINK5,

Anti-microbial MUC21,SLURP1) (DEFB1,S100A8,S100A9)
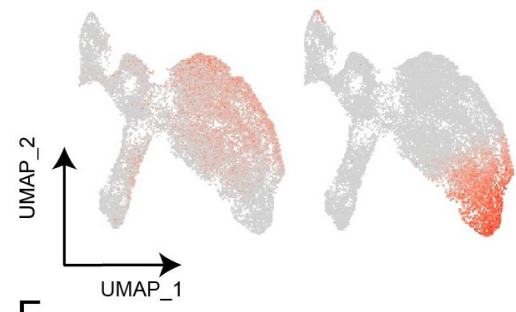

$\mathrm{F}$
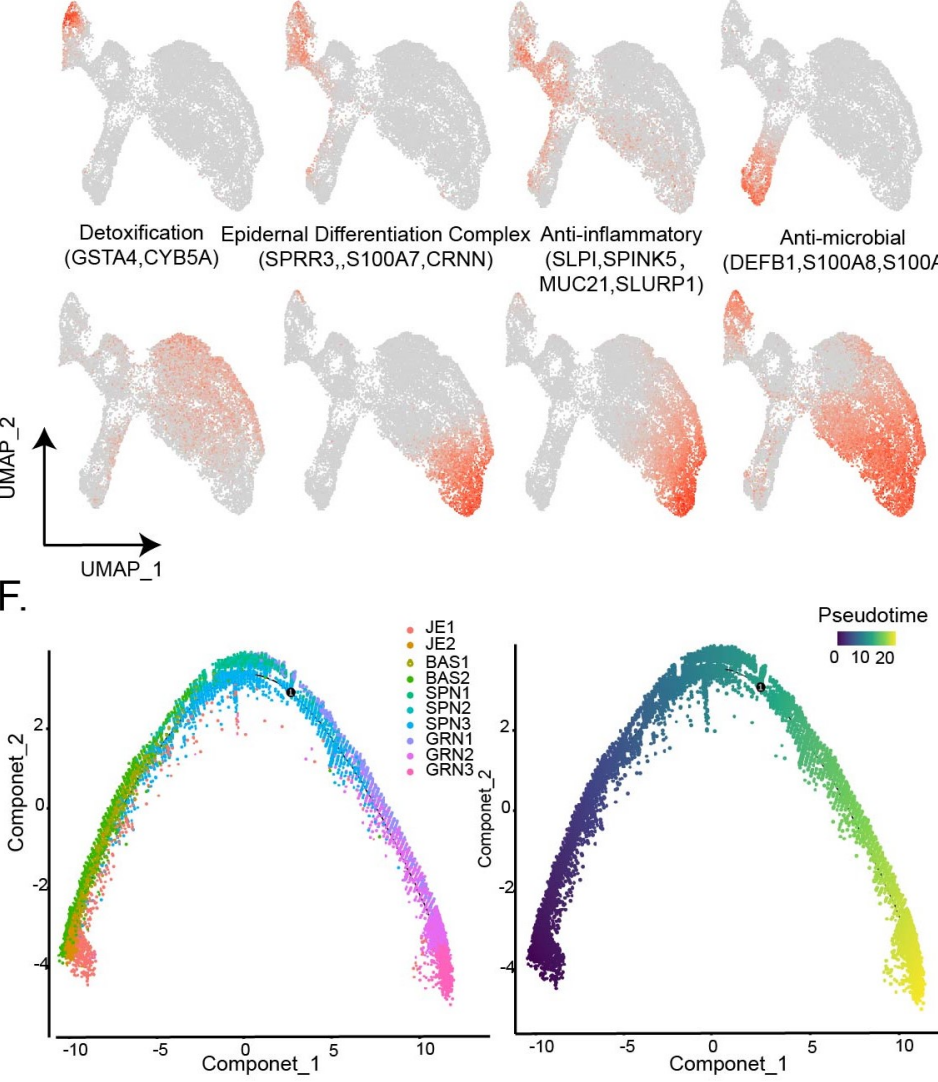

B.

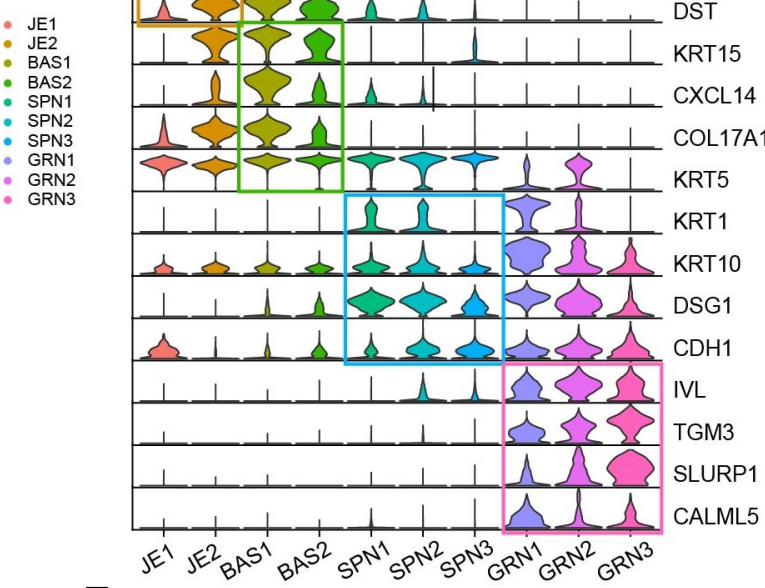

$E$

$\mathrm{G}$.
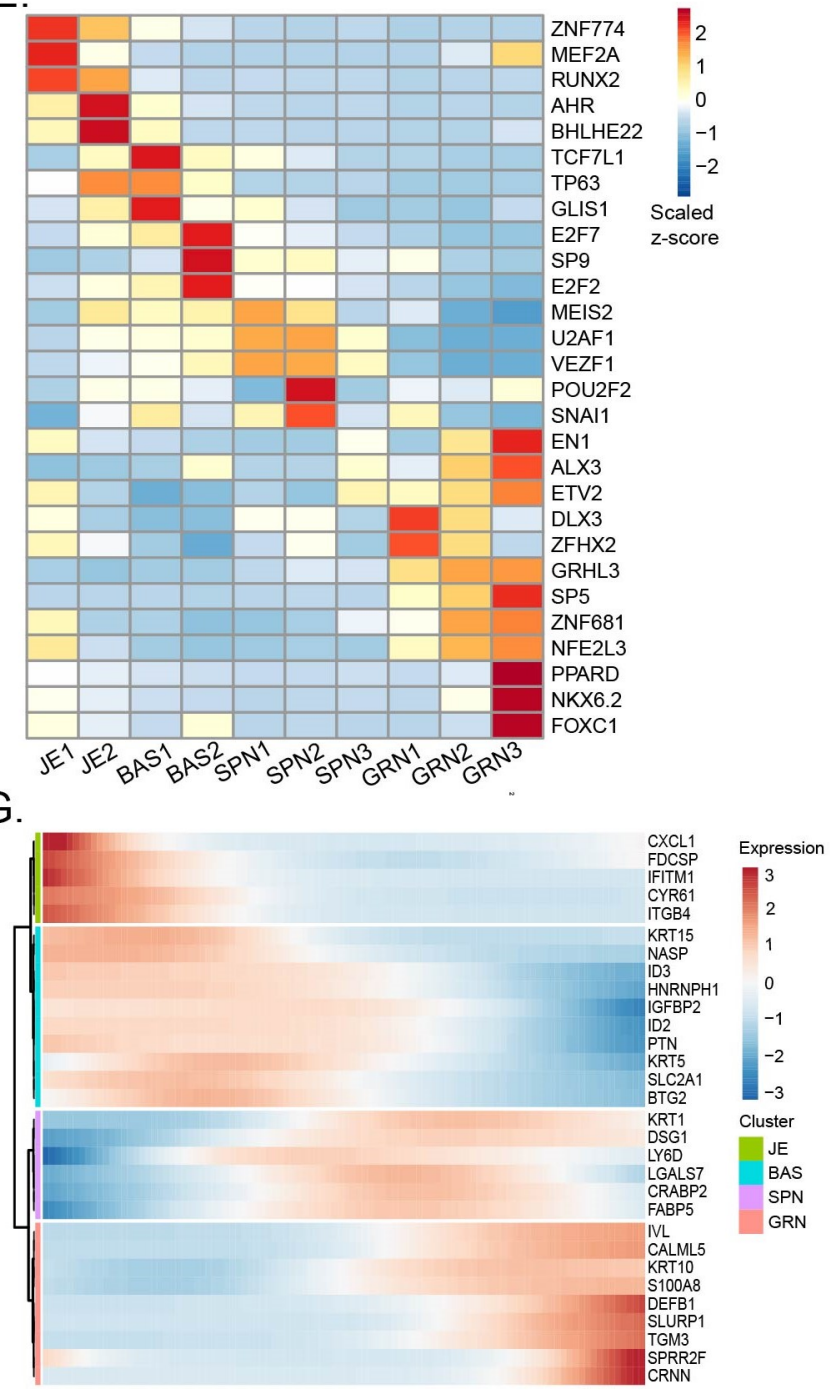
A.

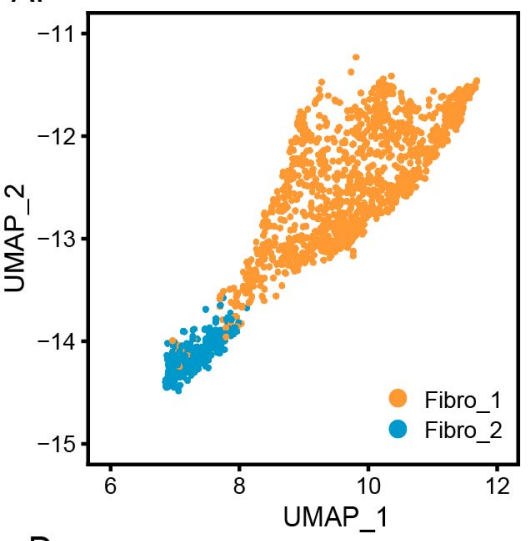

D.

Decorin

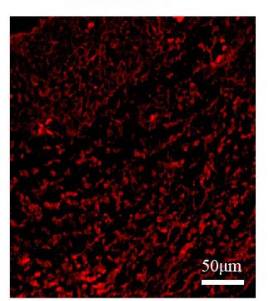

Osteoglycin

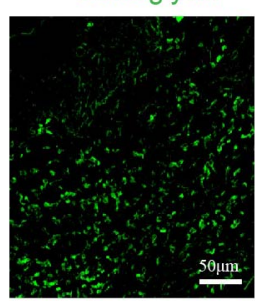

CXCL13

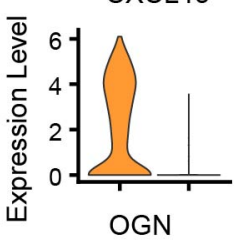

IL32

SFRP2

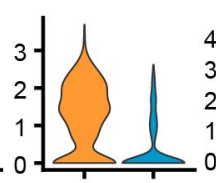

RUNX2
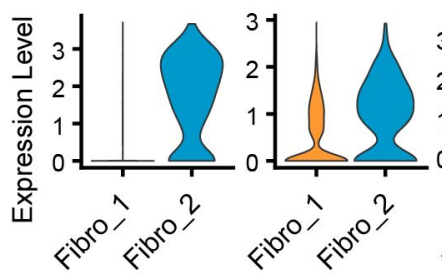

Fibro_1 vs Fibro_2

C.
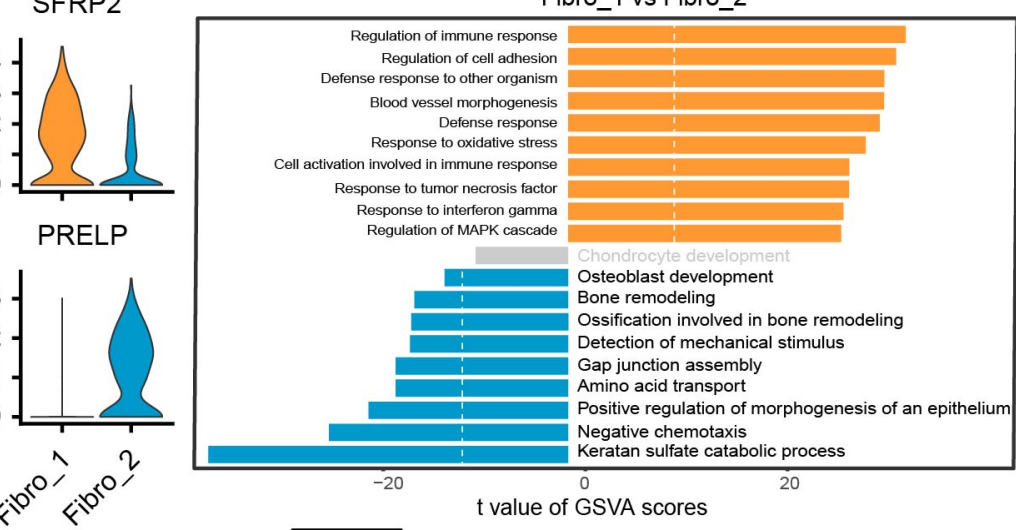

E.

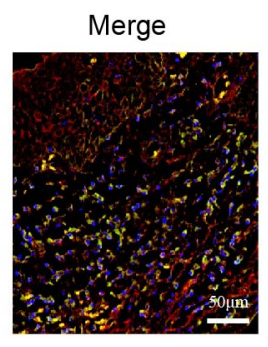

Nuclei

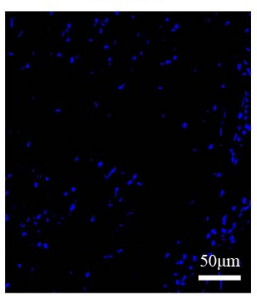

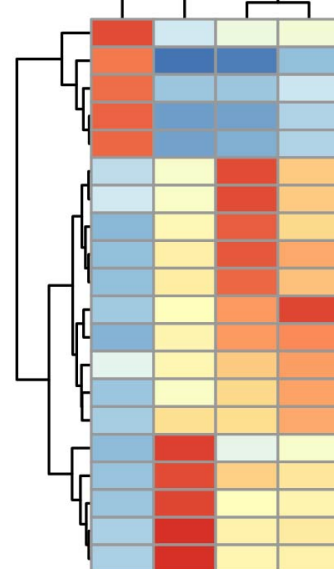

$\circ \quad \circ \quad \circ \quad 0 \quad$ Pearson

Epithelial.cell_KRT13.high.Adult.Esophagus2. Kerationcyte.Adult.Esophagus1.

Mucosal.aquamous.epithelial.cell.Adult.Esophagus1.

Epithelial.cell_KRT4.high.Adult.Esophagus1.

Epithelial.cell_KRT16.high.Adult.Esophagus1.

Stromal.cell_PTGDS.high.Adult.Esophagus2.

Fibroblast.Adult.Esophagus2.

Fibroblast_CXCL12.high.Adult.Artery1

Myofibroblast_POSTN.high.Adult.Artery1.

Fibroblast.Adult.Lung2.

PV1.Placenta_VentoTormo.

Smooth muscle.cell.Adult.Artery1.

Smooth.muscle.cell_CCL19.high.Adult.Trachea2.

Quiescent.stellate.cell.Adult.Pancreas_Baron

Smooth.muscle.cell.Adult.Lung1.

Endothelial.cell. Testis_Guo.

Endothelial.cell_VWF.high.Adult.Artery1.

Endothelial.cell VWF.high.Adult.Lung2.

Endo..m..Placenta_VentoTormo.

Endothelial.cell_SELE.high.Adult.Lung2.

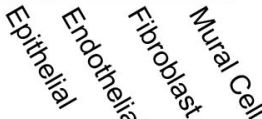


A.
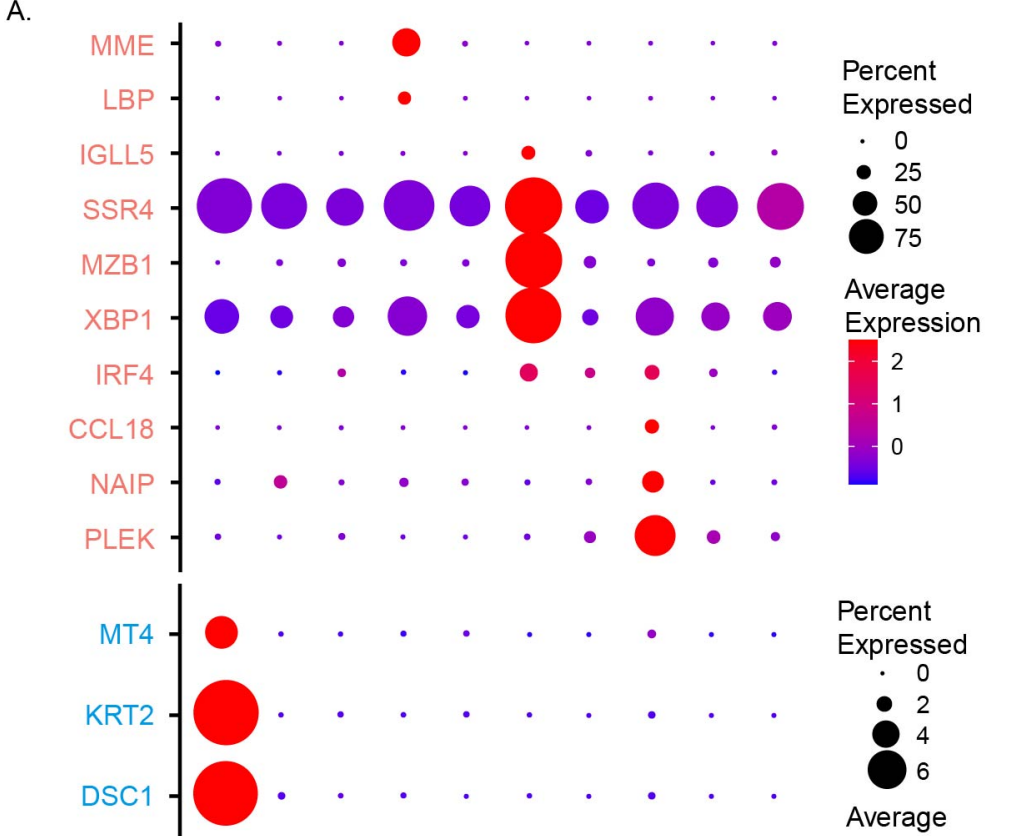

B.
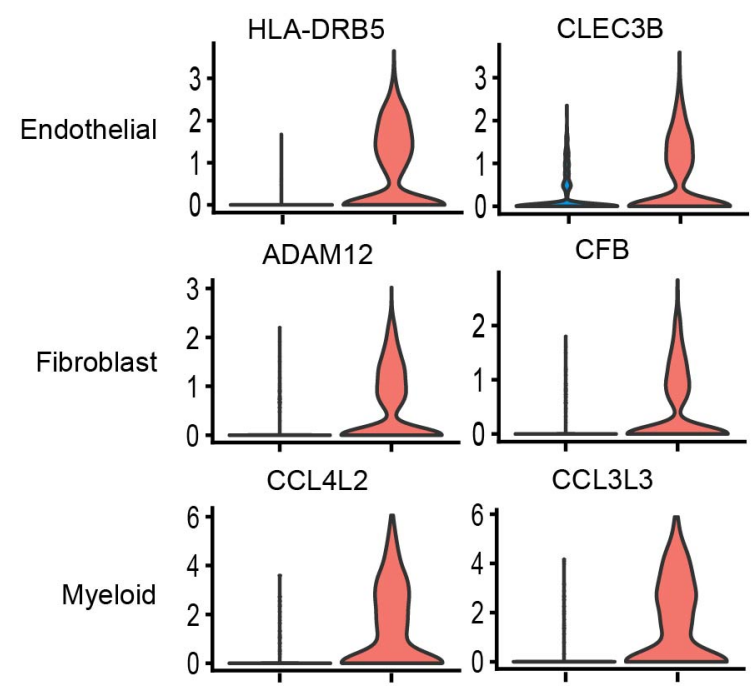

PDCD1

(CD4_CTLA4)

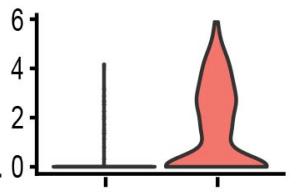

CTSW

(CD8_GZMK)

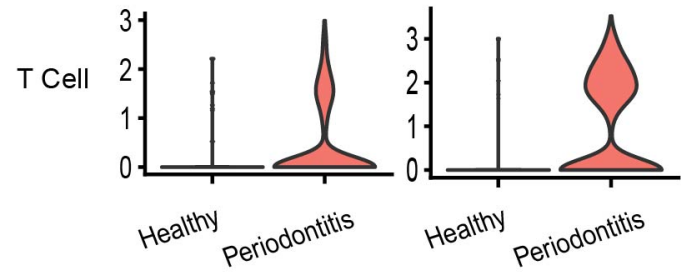

D.

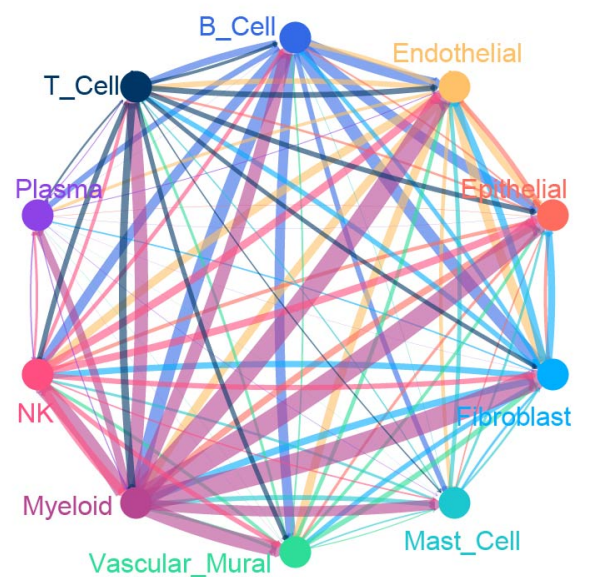

Vascular_Mural

Myeloid Plasma

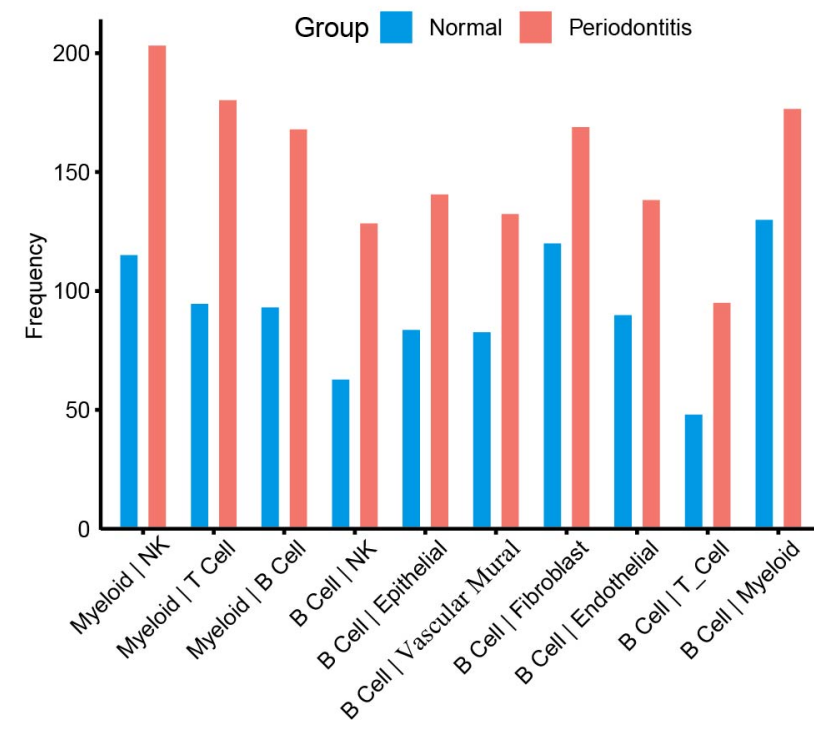

E.

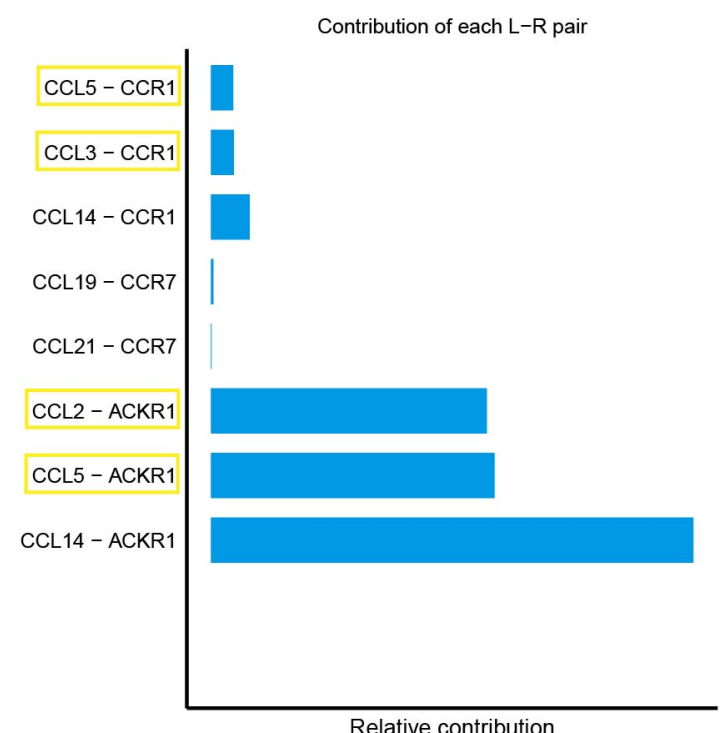

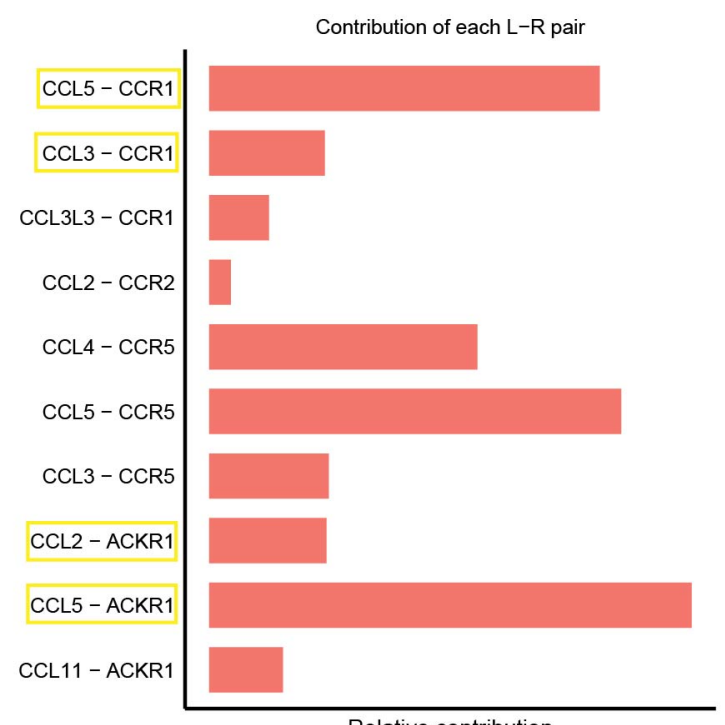

Group

Normal

Periodontitis 
A.

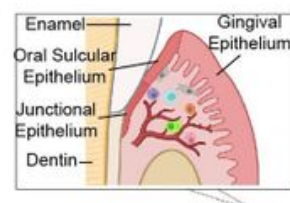

C.

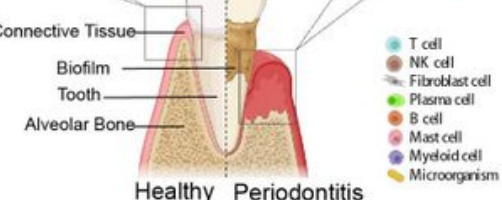

B.

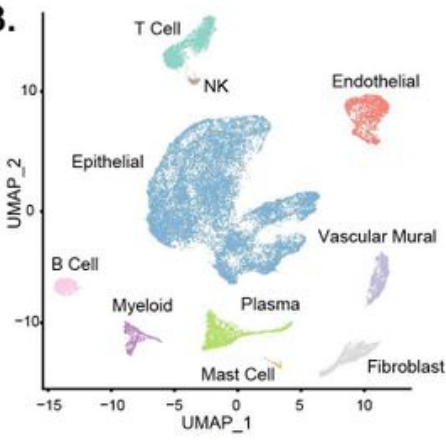

RGS5, PDGFRB, MCAM ACTA2, TAGLN
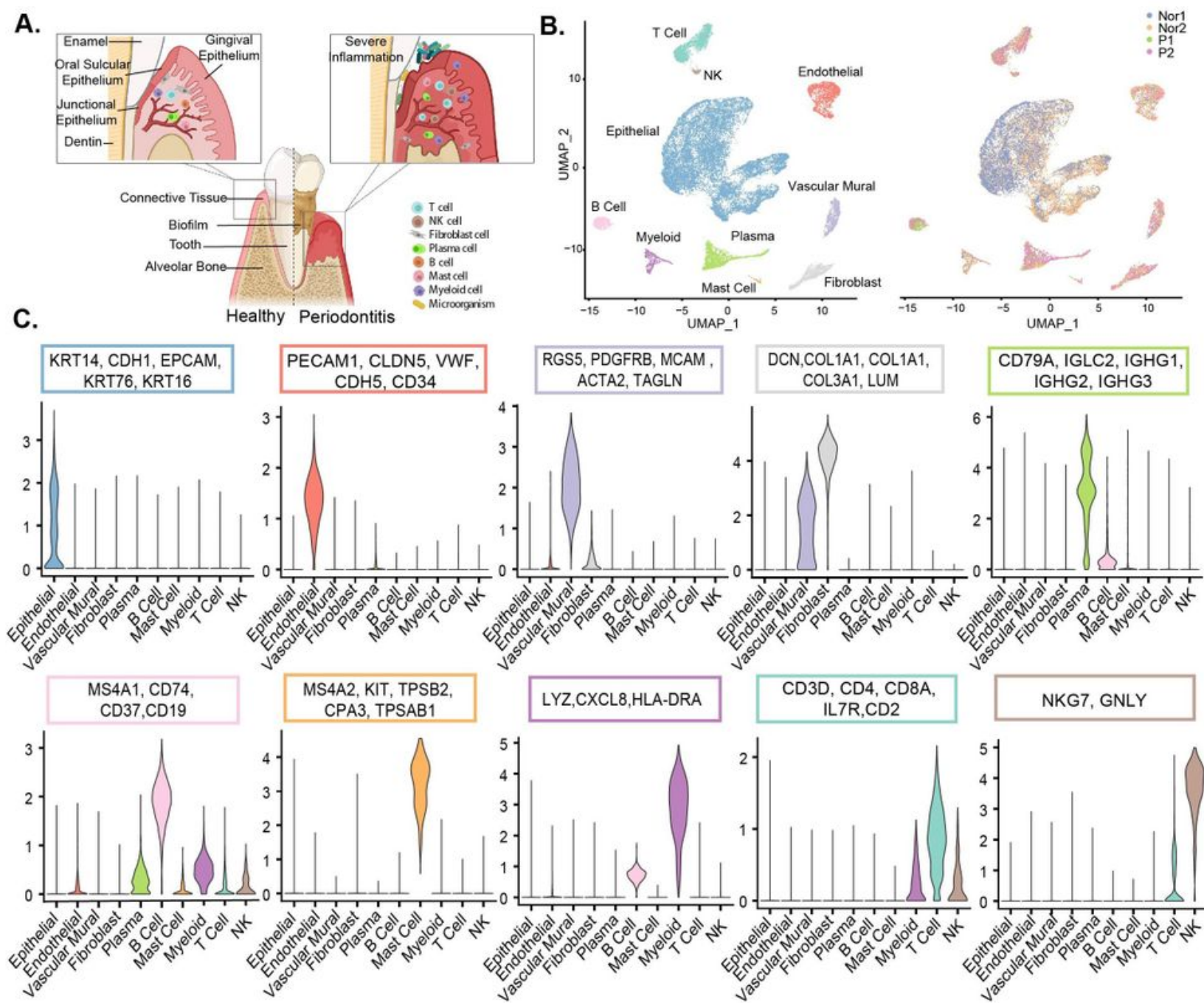

D.

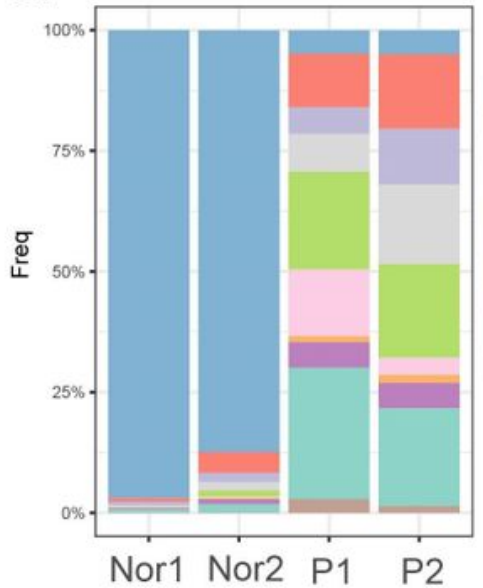

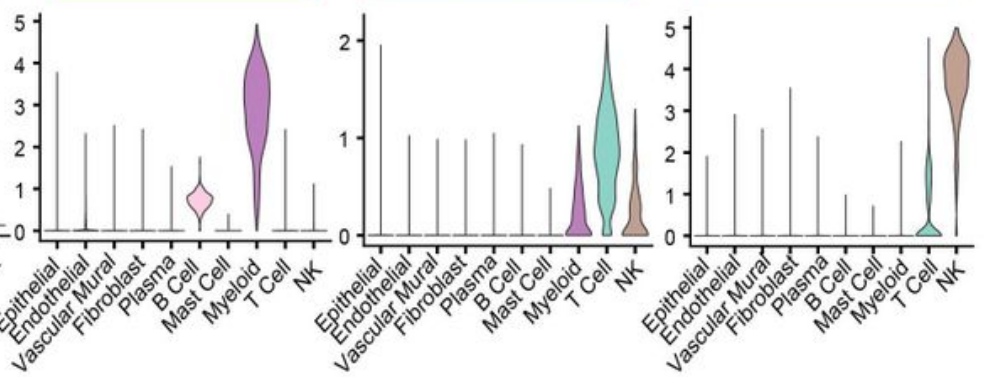

E.
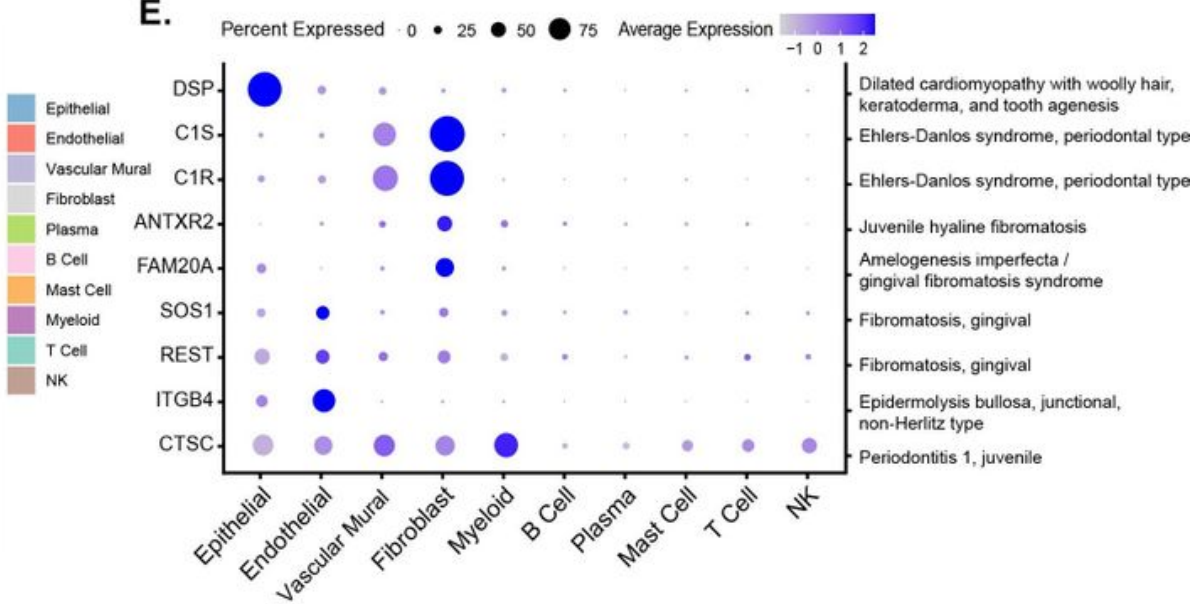

\section{Figure 1}

Overview of the clustering and annotation of the single-cell RNA sequencing data for gingival tissues. A. Schematic depicting anatomical regions analyzed in this study (The graph was created with BioRender.com). B. tSNE of the 29,967 cells profiled here, with each cell color-coded for (left to right): the 
corresponding patient and the associated cell type. C. Well-known markers scores for annotation of gingival cell types visualized by violin plot. D. Proportion of cell types from periodontitis and healthy conditions. (Nor1 and Nor2 are healthy donors; P1 and P2 are periodontitis patients) E. Dot plot depicting gene expression levels and percentage of cells expressing genes associated with periodontal disease according to the OMIM database.

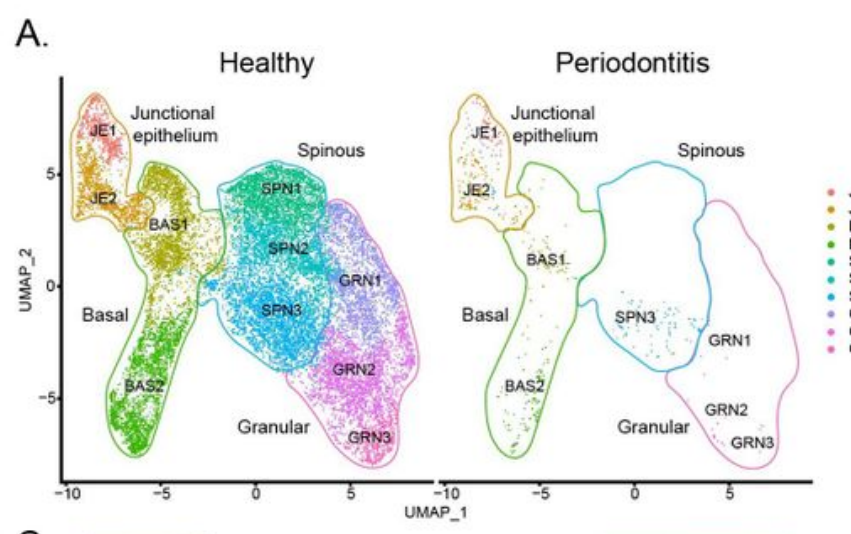

C.
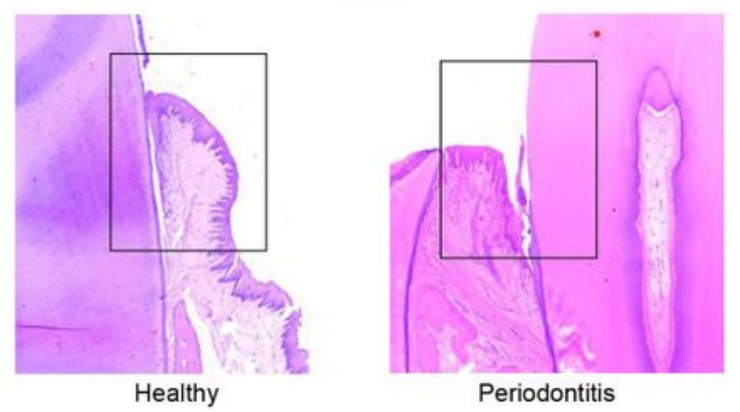

D.
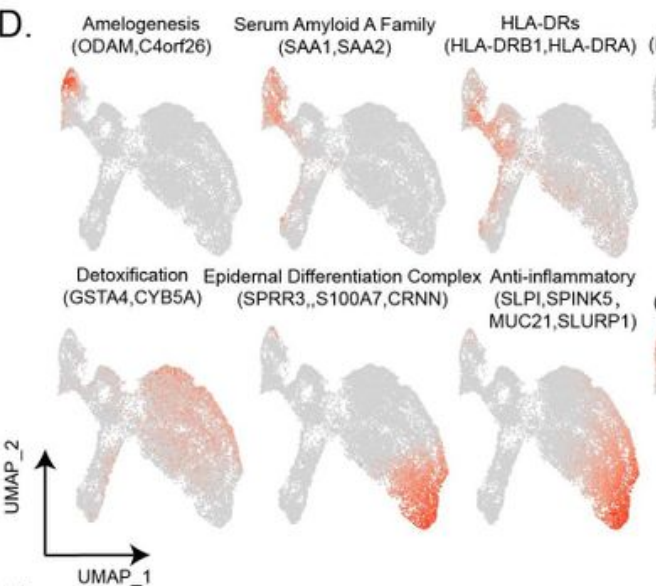

ernal Differentiation Complex
(SPRR3,_S100A7,CRNN)

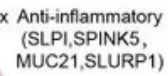

F.

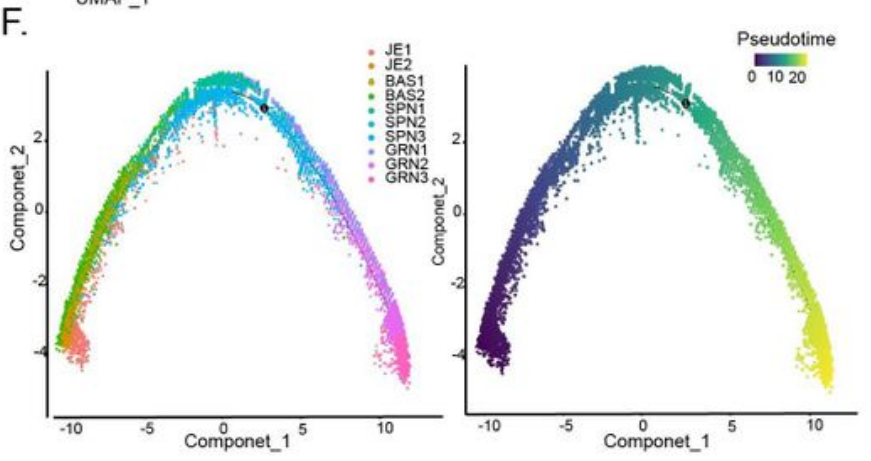

B.

E.
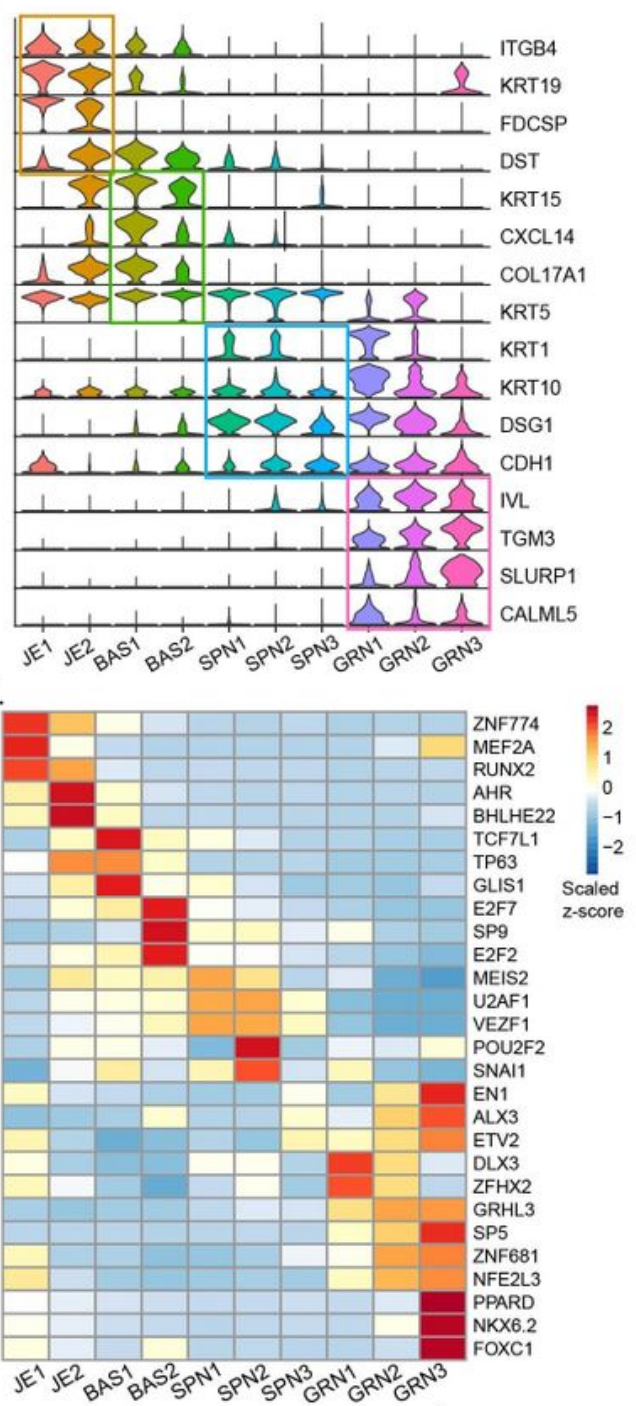

G.

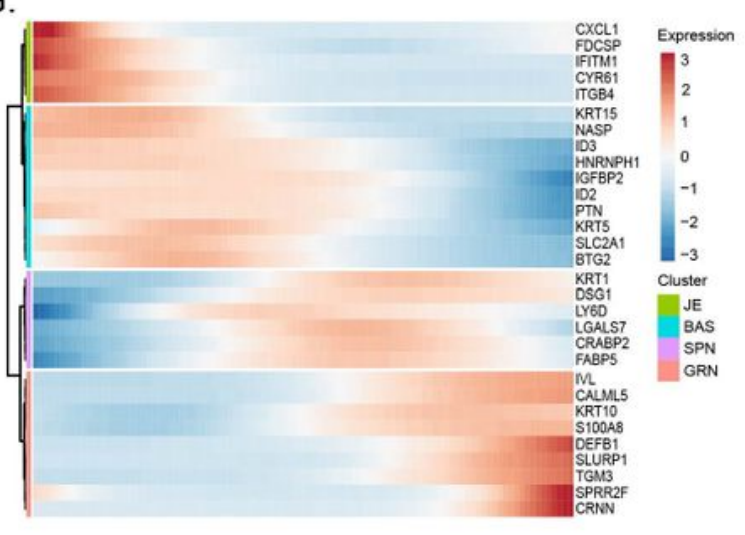


The heterogeneity and developmental trajectory of gingival epithelial. A. Visualization of human gingival epithelial cells in disease and healthy conditions separately. B. Markers for annotation of gingival epithelial cell types visualized by violin plot. C. Immunohistochemical showed the outermost layer of the epithelium experienced severe ruptures in patients. D. UMAP plots of epithelial cells, color-coded for functional molecules scores. E. Heatmap showing the activity of TFs in each epithelial cell subtype. The TF activity is scored using scaled AUCell. F. Monocle2-generated pseudotime trajectory of epithelial types imported from Seurat data, colored by cell-name designation (left) and colored in a gradient from dark to light yellow (right). G. Heatmap for clustering the 30 genes that vary as a function of pseudotime. The 30 genes were divided into four clusters, representing the genes at the beginning stage, the transitory stage, and the end stage of developmental trajectory, respectively.

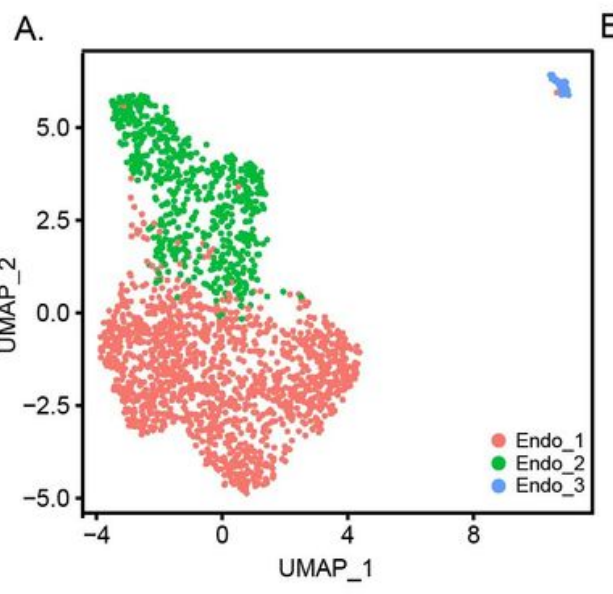

D. MHC Class II

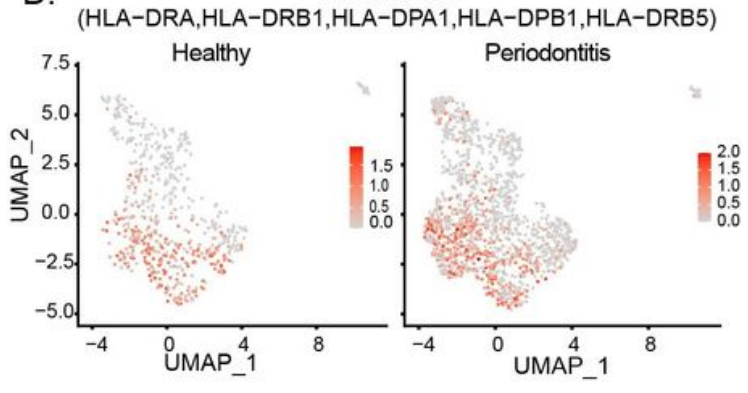

B.
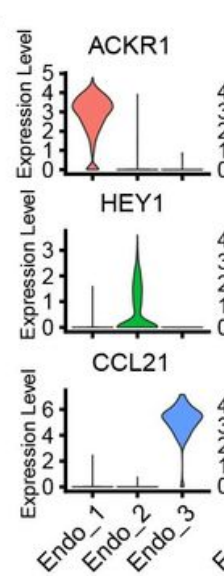

E.
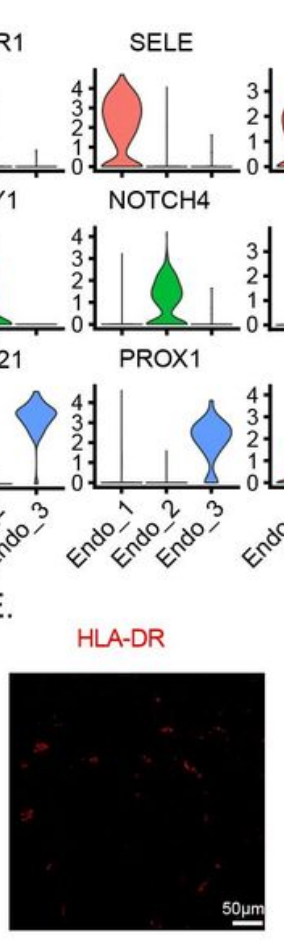
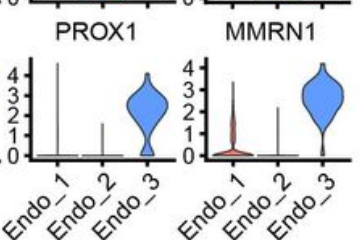

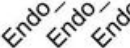

C.

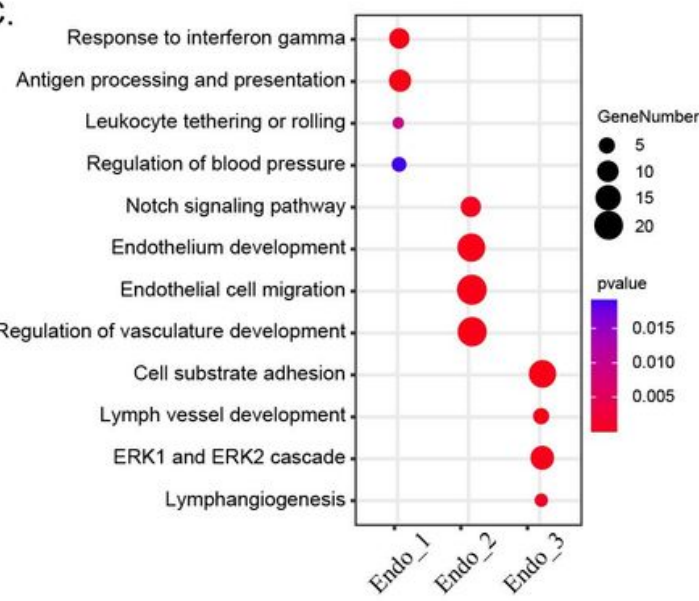

CD31

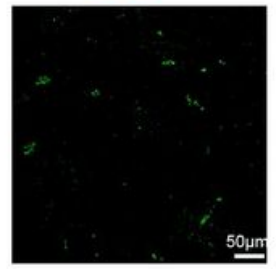

Nuclei

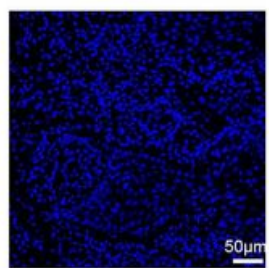

Merge

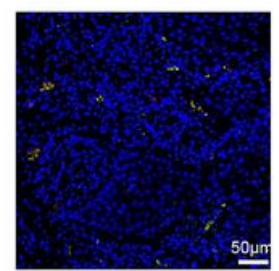

\section{Figure 3}

Three endothelial subclusters identified from the human gingival cells. A. UMAP visualization of three endothelial subclusters from human gingival cells. B. Violin plots showing the expression distribution of selected genes associated with functions in the endothelial clusters. C. Top gene ontology term enrichment for endothelial clusters. D. HLA-DRA showed expression specificity in the endothelial Endo_1. The gene expression levels are normalized and transformed as In (CPM/10). E. Immunofluorescent (IF) staining validation of endothelial subtypes. Red color showed the signal of HLA-DR staining; green color showed the signal of CD-31 staining (endothelial marker); and blue color showed DAPI staining. 
A.

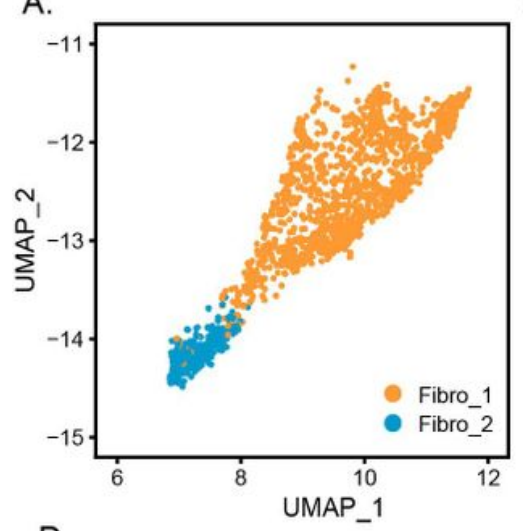

D.

Decorin

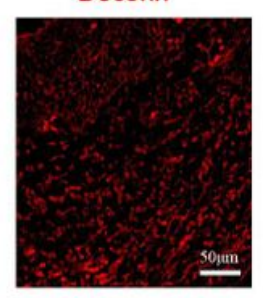

B.

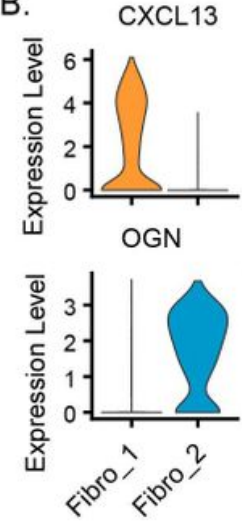

Nuclei

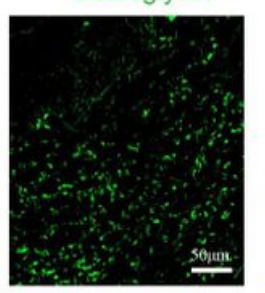

IL32

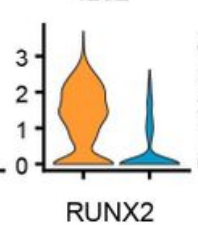

RUNX2
SFRP2

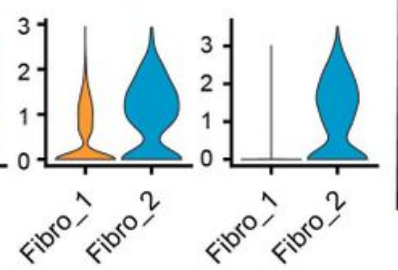

C.

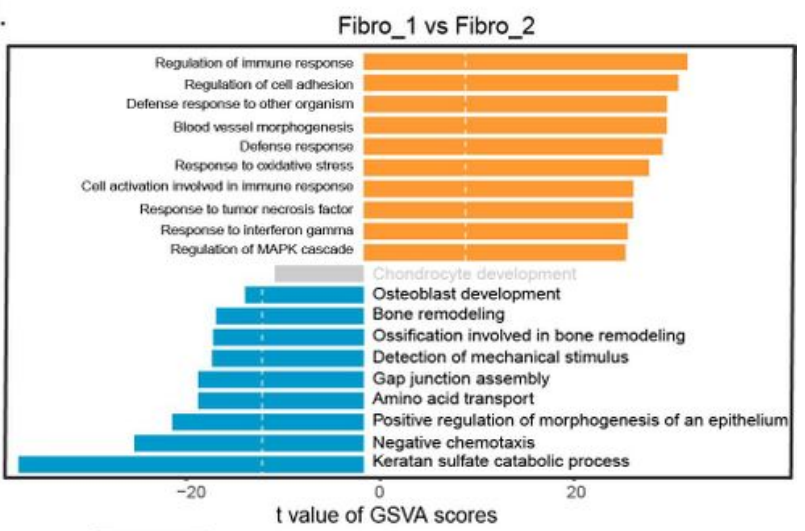

E.
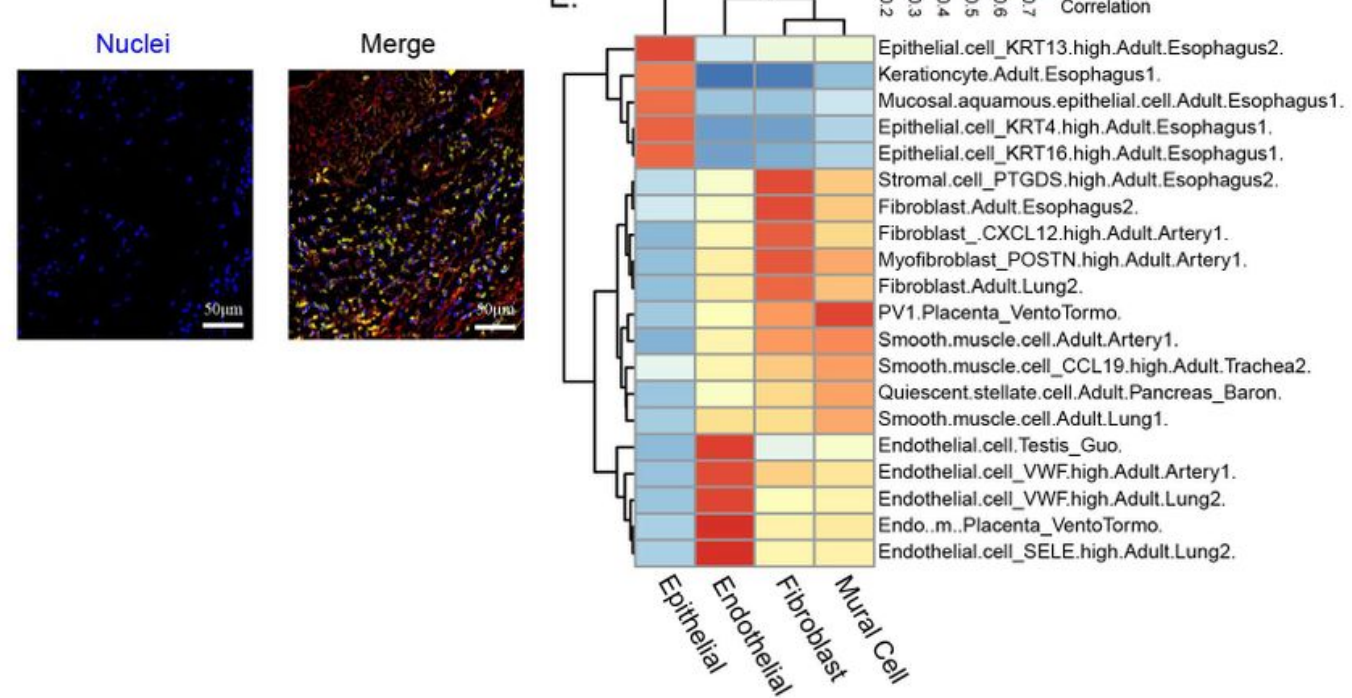

Figure 4

Two fibroblast subclusters identified from the human gingival cells. A. UMAP visualization of two fibroblast subclusters from human gingival cells. B. Violin plots showing the expression distribution of selected genes associated with functions in the endothelial clusters. The gene expression levels are normalized and transformed as In (CPM/10). C. Differences in pathway activities scored per cell by GSVA between fibro_1 and fibro_2. Shown are t values from a linear model, corrected for fibro_1. D. Immunofluorescent (IF) staining validation of fibroblast subtypes. The IF was performed on subepithelial region and connective tissue, respectively. Red color showed the signal of 727 Decorin staining (fibroblast marker); green color showed the signal of Osteoglycin (OGN) staining; and blue color showed DAPI staining. E. Application of scHCL analysis for non-immune cells. Each row represents one cell type in scHCL. Each column represents a cell cluster in our dataset. Pearson correlation coefficient was used to evaluate cell-type gene expression similarity. Red indicates a high correlation; blue indicates a low correlation. 
A.

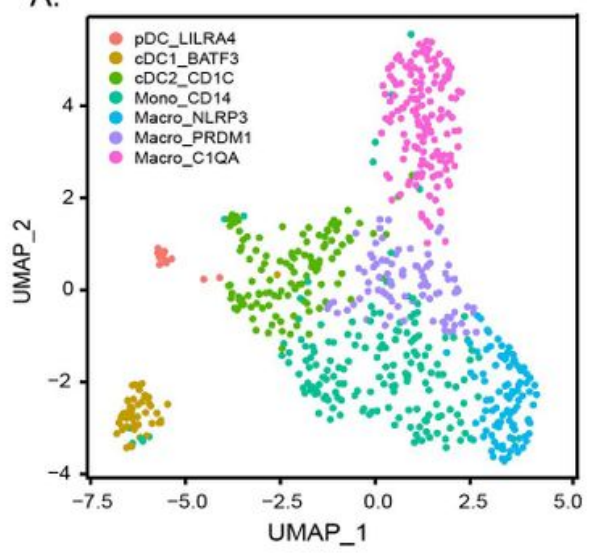

B.

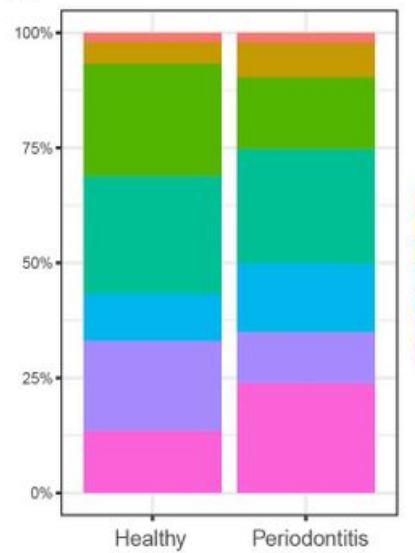

D. $\begin{array}{cc}\text { Phagocytosis } & \text { Angiogenesis } \\ \text { (MRC1,CD163,MERTK,C1QB) } & \text { (VEGFA,VCAN,CXCL8,ANGPTL4) }\end{array}$

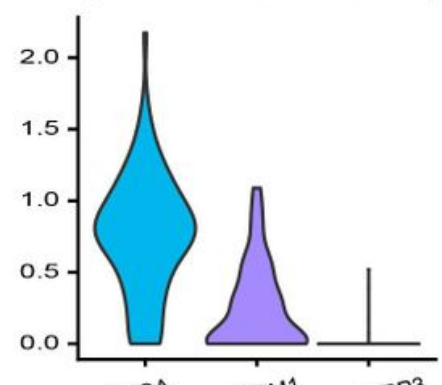
Macro_C1QA Macro_PRDM1 Macro_NLRP3 Macro_C1QA Macro_PRDM1 Macro NLRP3

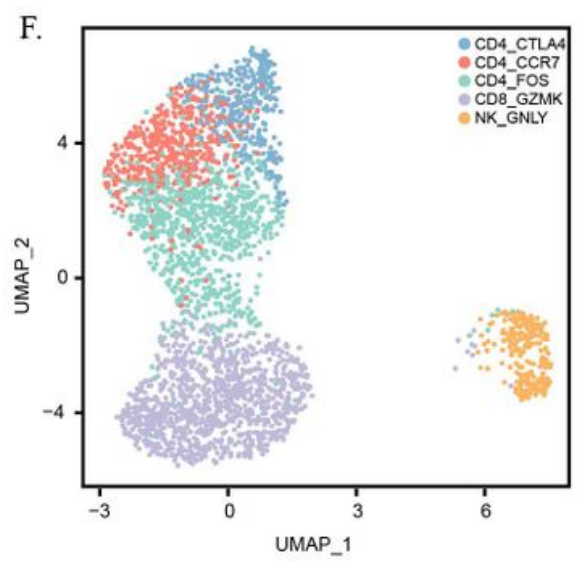

$\mathrm{G}$.

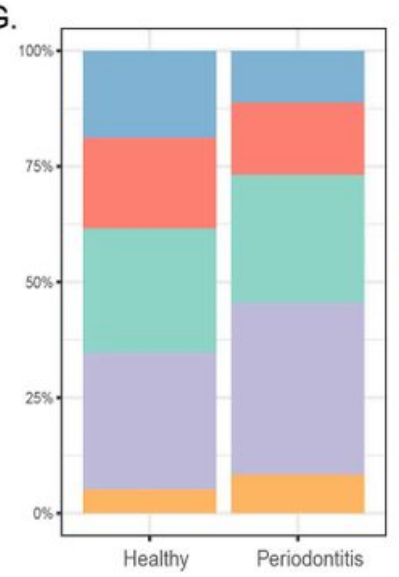

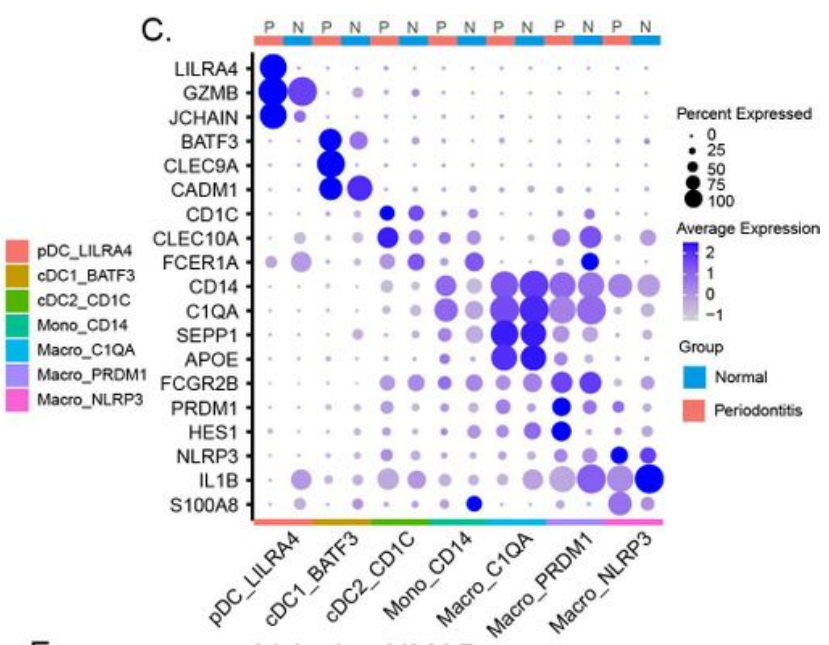

E.

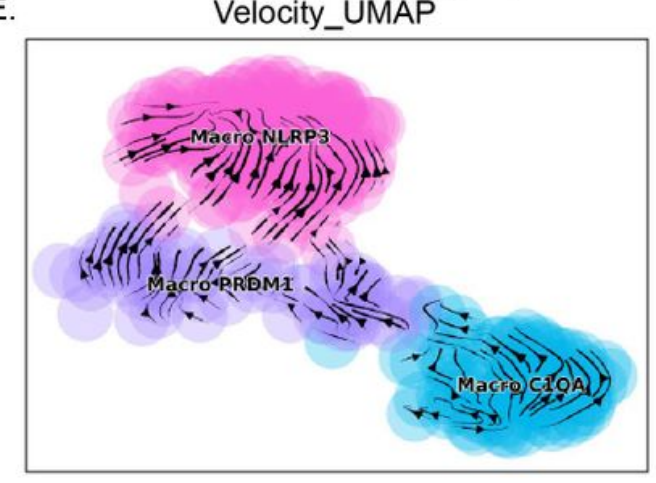

H.

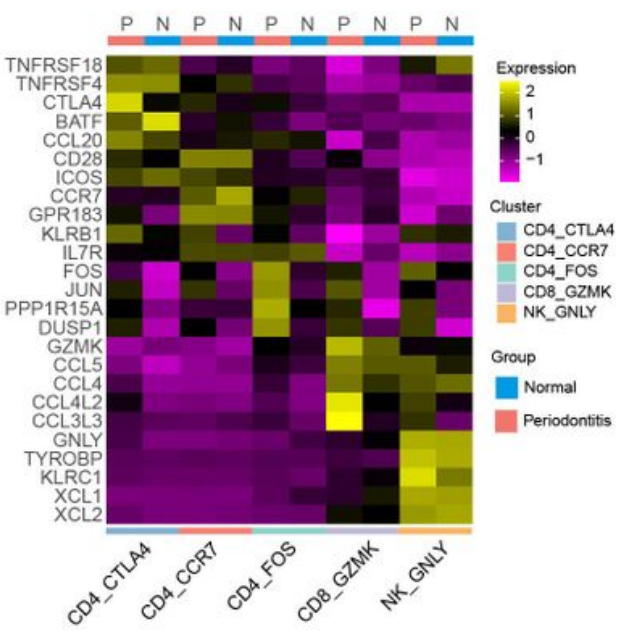

\section{Figure 5}

The heterogeneity of myeloid and T cells. A. UMAP visualization of seven myeloid clusters from human gingival cells. B. Relative proportion of myeloid cell subsets from different origin (healthy $=2$ and periodontitis=2). C. Bubble heatmap showing marker genes across seven myeloid clusters from (A). Dot size indicates fraction of expressing cells, colored according to z-score normalized expression levels. D. Violin plots showing the expression of angiogenesis- and phagocytosis-related genes in three macrophages clusters. The gene expression levels are normalized and transformed as In (CPM/10). E. Velocity field projected onto the UMAP plot of three macrophages (arrows represent average velocity). F. UMAP visualization of seven myeloid clusters from human gingival cells. G. Relative proportion of T cell 
subsets from different origin (healthy $=2$ and periodontitis=2). H. Heatmap of $\mathrm{T}$ cell top 5 DEGs in each cell cluster in healthy and periodontitis.

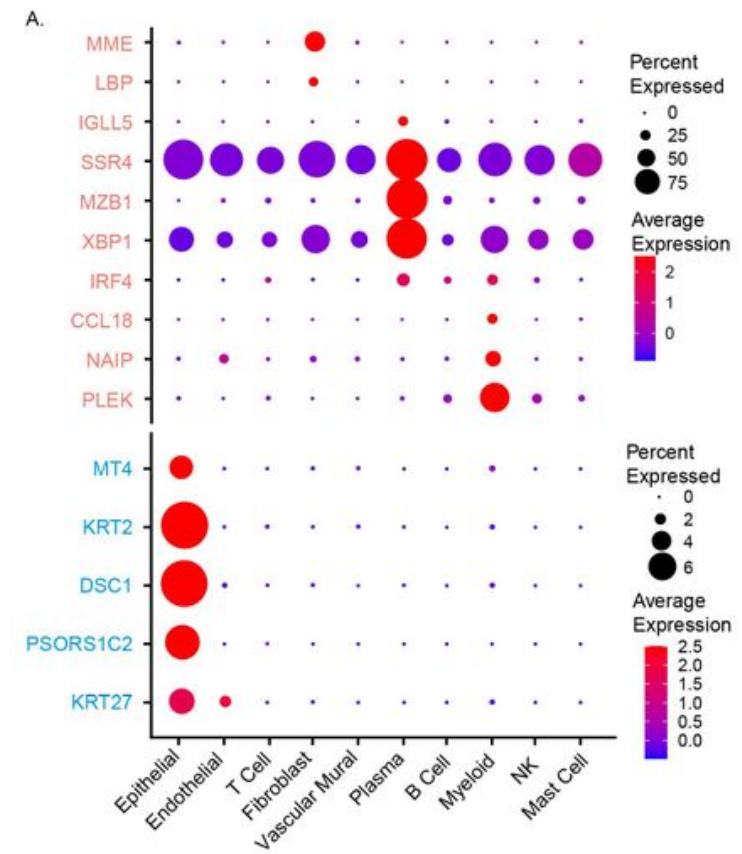

B.
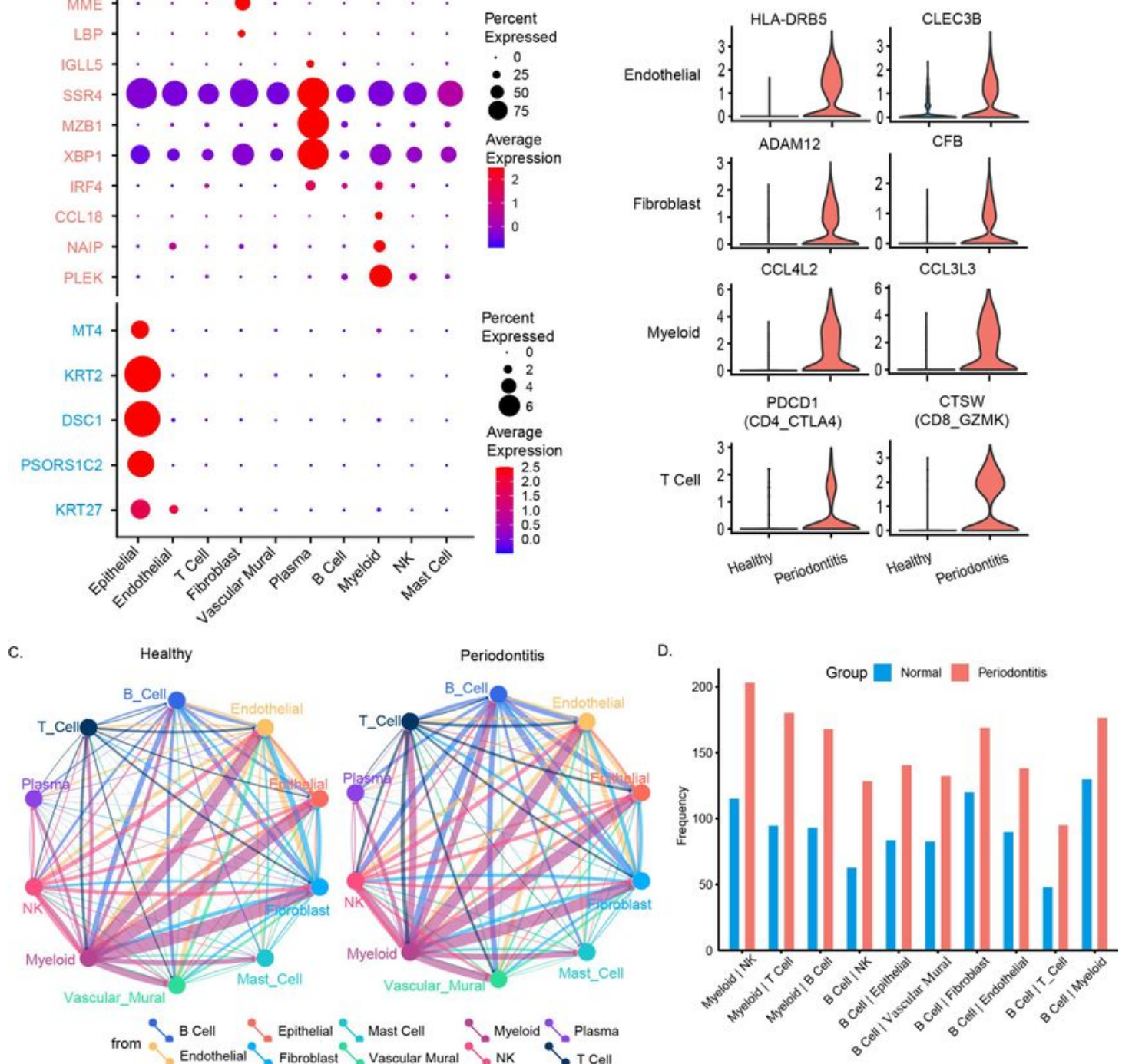

E.
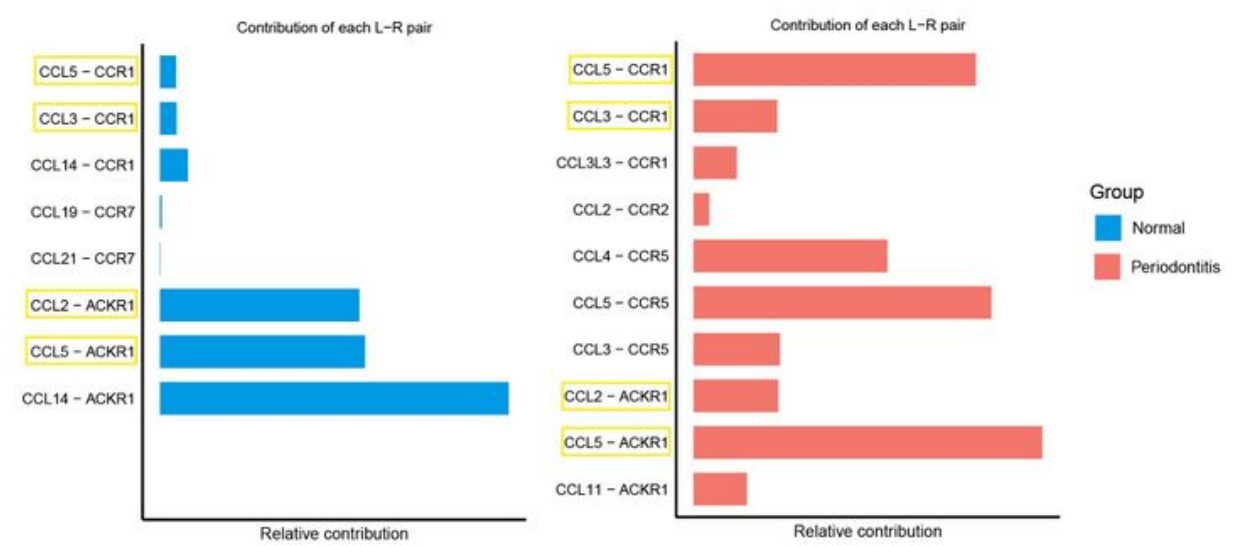

Figure 6

The difference among gingival cell types in healthy and periodontitis conditions. A. Bubble heatmap showing reported genes associated diseases clusters from (Figure 1B). Rose stands for reported increase in periodontitis, and turquoise stands for reported decrease in periodontitis. Dot size indicates fraction of 
expressing cells, colored according to z-score normalized expression levels. B. Violin plots showing the expression of gene specific increasing in different clusters of periodontitis. The gene expression levels are normalized and transformed as In (CPM/10). C. Cell-cell interaction network in healthy and periodontitis condition. Colors and widths of edges represent number of interaction pairs between cell types. D. The top 10 cell-cell interactions with the most significant increase in patients than healthy. The relative contribution of receptor-ligand in CCL pathway in normal and periodontitis. Yellow indicates that the common receptor-ligand is present in both the patient and the normal.

\section{Supplementary Files}

This is a list of supplementary files associated with this preprint. Click to download.

- Table1S13.pdf

- supplementtable2OMIM.xIsx

- supplementtable3scenicTF.xlsx

- Supplementtable4monoclegenes.csv 